\title{
Optimal approximate quantum error correction for quantum metrology
}

\author{
Sisi Zhou (1) and Liang Jiang (1) \\ Departments of Applied Physics and Physics, Yale University, New Haven, Connecticut 06511, USA; \\ Yale Quantum Institute, Yale University, New Haven, Connecticut 06511, USA; \\ and Pritzker School of Molecular Engineering, The University of Chicago, Chicago, Illinois 60637, USA
}

(Received 3 November 2019; accepted 5 February 2020; published 2 March 2020)

\begin{abstract}
For a generic set of Markovian noise models, the estimation precision of a parameter associated with the Hamiltonian is limited by the $1 / \sqrt{t}$ scaling, where $t$ is the total probing time, in which case the maximal possible quantum improvement in the asymptotic limit of large $t$ is restricted to a constant factor. However, situations arise where the constant factor improvement could be significant, yet no effective quantum strategies are known. Here we propose an optimal approximate quantum error correction (AQEC) strategy asymptotically saturating the precision lower bound in the most general adaptive parameter estimation scheme, where arbitrary and frequent quantum controls are allowed. We also provide an efficient numerical algorithm finding the optimal code. Finally, we consider highly biased noise and show that using the optimal AQEC strategy, strong noises are fully corrected, while the estimation precision depends only on the strength of weak noises in the limiting case.
\end{abstract}

DOI: 10.1103/PhysRevResearch.2.013235

\section{INTRODUCTION}

Quantum metrology is one of the most important state-ofthe-art quantum technologies, studying the precision limit of parameter estimation in quantum systems [1-6]. The task involves preparing a suitable initial state of the system, allowing it to evolve under quantum controls for a specific time, performing a suitable measurement, and inferring the value of the unknown system parameter from the measurement outcome. To enhance the estimation precision, a variety of quantum strategies has been proposed, such as squeezing the initial state [7-12], optimizing the probing time [13], monitoring the environment [14-16], exploiting non-Markovian effects [17-19], optimizing the control Hamiltonian [20-22], and quantum error correction [23-36].

Quantum mechanics places a fundamental limit on estimation precision, i.e., the Heisenberg limit (HL), where the estimation precision scales like $1 / N$ for $N$ probes or, equivalently, $1 / t$ for a total probing time $t$. In the noiseless case, the HL is achievable using the maximally entangled state among probes $[1,37]$. In practice, decoherence plays an indispensable role. Under many typical noise models, the estimation precision will follow the standard quantum limit (SQL) with scaling $1 / \sqrt{N}$ (or $1 / \sqrt{t}$ ) [29-31,38-43], the same as the central limit theorem scaling using classical strategies. Nevertheless, the superiority of quantum strategies over classical strategies by a constant-factor improvement, as opposed to a scaling improvement, was proven in several cases $[11,39,42]$. There

\footnotetext{
*Corresponding author: sisi.zhou@yale.edu

Published by the American Physical Society under the terms of the Creative Commons Attribution 4.0 International license. Further distribution of this work must maintain attribution to the author(s) and the published article's title, journal citation, and DOI.
}

were also situations where the HL is achievable using quantum strategies even in the presence of noise [16,31].

Due to the difficulty in obtaining the exact precision limits for general noise models using different quantum strategies, several asymptotical lower bounds were proposed [29-31,3844]. For example, the channel simulation method was used to prove the SQL lower bound for programmable channels [40-42]. A necessary and sufficient condition of achieving the HL under Markovian noise - the "Hamiltonian not in the Lindblad span" (HNLS) condition-was established using the channel extension method [29-31]. Although these bounds were successful at showing the scaling limit of quantum strategies, only in several special cases, the saturability of these lower bounds was established, e.g., for dephasing and erasure noise [42] and for teleportation-covariant channels as a special type of programmable channels $[45,46]$. A saturability statement of the SQL lower bound under general noise models and an efficient algorithm solving the optimal strategy remain missing up to the present day.

We address both of these open questions in this work. Here we consider parameter estimation under general Markovian noise using the most general adaptive sequential strategy (see Fig. 1). We propose an approximate quantum error correction (AQEC) strategy saturating the SQL lower bound of precision (asymptotically) and an efficient numerical algorithm solving the optimal AQEC codes for different noises. The saturability of the precision lower bound that we prove here not only answers an important question in quantum metrology theory, but also paves the way for identifying the optimal quantum strategies in future experiments.

Quantum error correction (QEC) [47] was first shown to be useful in quantum metrology in a typical scenario where the dephasing noise in a qubit probe is corrected by QEC, while the $X$ (the Pauli- $X$ operator) signal remains intact [23-26]. Later on, it was proven that for arbitrary Markovian noise [48-50], the HL is achievable using the sequential QEC 


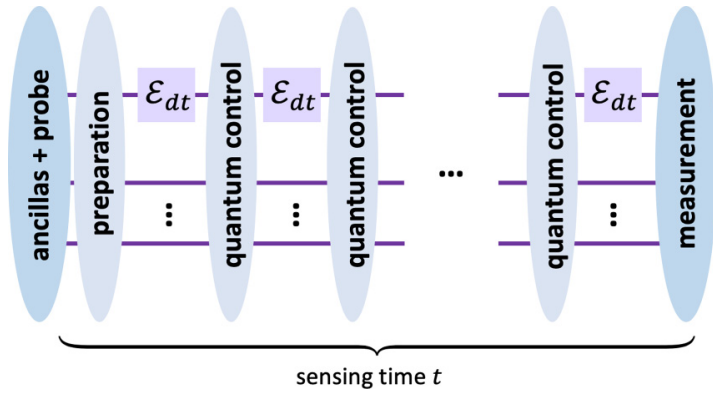

FIG. 1. The most general adaptive sequential strategy where one probe sequentially senses the parameter for time $t$, with quantum controls [arbitrary completely positive and trace-preserving (CPTP) maps] applied every $d t$ and an arbitrary number of noiseless ancillas available. $\mathcal{E}_{d t}(\rho)=\rho+\left(-i[\omega H, \rho]+\sum_{i=1}^{r} L_{i} \rho L_{i}^{\dagger}-\right.$ $\left.\frac{1}{2}\left\{L_{i}^{\dagger} L_{i}, \rho\right\}\right) d t+O\left(d t^{2}\right)$ describes the evolution of the probe in an infinitesimally small time interval $d t$.

strategy if HNLS is satisfied [30,31]. In practice, HNLS is often violated, for example, when sensing any single-qubit signal under depolarizing noise, and the estimation precision follows the SQL. Standard QEC strategies would be useless in this case, as it will eliminate both the signal and the noise simultaneously. However, by performing AQEC where the noise is only partially corrected, the estimation precision could still be improved in some cases [29,33]. Here we show that the AQEC strategy could be used in general to achieve the optimal precision limit for any Markovian noise.

In this paper, we first review the SQL precision lower bound under Markovian noise when HNLS is violated. Then we describe our AQEC strategy consisting of both a twodimensional AQEC code and an optimal recovery channel. This allows the original quantum channel to be reduced to an effective channel where a $Z$ (the Pauli- $Z$ operator) signal was sensed under dephasing noise-a special case where the precision lower bound was known to be saturable $[11,39,42]$. Finally, we optimize the achievable precision over all possible AQEC codes, which coincides with the precision lower bound, completing the proof.

\section{PRECISION LOWER BOUND}

We assume the evolution of the quantum system is described by the following quantum master equation [48-50]:

$$
\frac{d \rho}{d t}=-i[\omega H, \rho]+\sum_{i=1}^{r}\left(L_{i} \rho L_{i}^{\dagger}-\frac{1}{2}\left\{L_{i}^{\dagger} L_{i}, \rho\right\}\right),
$$

where $\omega$ is the unknown parameter, $\rho \in \mathcal{H}_{S} \otimes \mathcal{H}_{A}, \mathcal{H}_{S}$ is the probe space $H$ and $\left\{L_{i}\right\}_{i=1}^{r}$ act on $\left(H, L_{i}\right.$ are shorthand for $H \otimes \mathbb{1}, L_{i} \otimes \mathbb{1}$, respectively), and $\mathcal{H}_{A}$ is the noiseless ancillary space (see Fig. 1). We assume $I,\left\{L_{i}\right\}_{i=1}^{r}$ are linearly independent, $\operatorname{dim} \mathcal{H}_{S}=d$ and $\operatorname{dim} \mathcal{H}_{A}=2 d$. The Lindblad span associated with Eq. (1) is $\mathcal{S}=\operatorname{span}\left\{\mathbb{1}, L_{i}, L_{i}^{\dagger}, L_{i}^{\dagger} L_{j}, \forall i, j\right\}$, where $\operatorname{span}\{\cdot\}$ denotes the real linear subspace of Hermitian operators spanned by $\{\cdot\}$. According to the quantum CramérRao bound [51-54], the standard deviation $\delta \omega$ of the $\omega$ estimator is bounded by $\delta \omega \geqslant\left[N_{\text {expr }} F(t)\right]^{-1 / 2}$, where $N_{\text {expr }}$ is the number of experiments and $F(t)$ is the so-called quantum Fisher information (QFI) as a function of the final state $\rho(t)$.
The bound is asymptotically saturable using the maximum likelihood estimator as $N_{\text {expr }}$ goes to infinity [55,56]. Therefore, finding the optimal sequential strategy boils down to maximizing $F(t)$ over all input states and quantum controls. For an input state $|\psi\rangle$ evolving noiselessly under Hamiltonian $\omega H, F(t)=4 t^{2}\left[\left\langle\psi\left|H^{2}\right| \psi\right\rangle-(\langle\psi|H| \psi\rangle)^{2}\right]$ and $\delta \omega \propto 1 / t$ follows the HL. In the noisy case, it was proven that the HL is achievable if and only if $H \notin \mathcal{S}$ (the HNLS condition) and there exists a QEC strategy achieving the HL [30,31].

The HNLS condition usually holds when the noise has a special structure, e.g., rank-one noise [29] or spatially correlated noise $[32,33]$. For generic noise, however, the HNLS condition is often violated. In this paper, we focus on the latter situation where $H \in \mathcal{S}$ and the QFI follows the SQL [30,31],

$$
F(t) \leqslant 4 t \min _{h, \mathbf{h}, \mathfrak{h} \mid \beta=0}\|\alpha\|,
$$

where $\|\cdot\|$ is the operator norm of a matrix, $h \in \mathbb{R}, \mathbf{h} \in \mathbb{C}^{r}$, $\mathfrak{h} \in \mathbb{C}^{r \times r}$ is Hermitian,

$$
\begin{gathered}
\alpha=(\mathbf{h} \mathbb{1}+\mathfrak{h} \mathbf{L})^{\dagger}(\mathbf{h} \mathbb{1}+\mathfrak{h} \mathbf{L}), \\
\beta=H+h \mathbb{1}+\mathbf{h}^{\dagger} \mathbf{L}+\mathbf{L}^{\dagger} \mathbf{h}+\mathbf{L}^{\dagger} \mathfrak{h} \mathbf{L},
\end{gathered}
$$

where $\mathbf{L}:=\left(L_{1}, L_{2}, \ldots, L_{r}\right)^{T}$ and $\mathbf{h} \mathbb{1}:=\left(\mathbf{h}_{1} \mathbb{1}, \ldots, \mathbf{h}_{r} \mathbb{1}\right)^{T}$. Here we introduce an AQEC strategy which (asymptotically) saturates the QFI upper bound up to an arbitrarily small error under arbitrary Markovian noise. That is, for any small $\delta>0$, there exists an AQEC strategy such that

$$
\mathfrak{F}:=\sup _{t>0} \frac{F(t)}{t}>4 \min _{h, \mathbf{h}, \mathfrak{h} \mid \beta=0}\|\alpha\|-\delta,
$$

where we define the normalized QFI $\mathfrak{F}$ as the objective function that we maximize. The upper bound is saturated asymptotically in the sense that $\lim _{t \rightarrow \infty} F(t) / t=\mathfrak{F}$ because, for any $t_{0}$ such that $F\left(t_{0}\right) / t_{0} \approx \mathfrak{F}$, we can always find a sensing strategy such that $t=k t_{0}$ and $F\left(k t_{0}\right) /\left(k t_{0}\right)=F\left(t_{0}\right) / t_{0}$ for all integers $k$ by measuring and renewing the probing state every constant time $t_{0}$. Then, $\lim _{t \rightarrow \infty} F(t) / t \geqslant \lim _{k \rightarrow \infty} F\left(k t_{0}\right) /$ $\left(k t_{0}\right) \approx \mathfrak{F}$.

\section{APPROXIMATE QUANTUM ERROR CORRECTION}

Here we propose a set of AQEC codes for quantum metrology and show that the effective channel under fast AQEC is an effective qubit dephasing channel in the logical space. In this way, identifying the optimal recovery channel for quantum metrology is equivalent to minimizing the noise rate of the dephasing channel where a closed-form solution exists, as opposed to general AQEC scenarios where many known AQEC recovery channels are only suboptimal [57-62].

Let $P$ be the projection onto the code space $\left|0_{\mathrm{L}}\right\rangle\left\langle 0_{\mathrm{L}}\right|+$ $\left|1_{\mathrm{L}}\right\rangle\left\langle 1_{\mathrm{L}}\right|$, where $\left|0_{\mathrm{L}}\right\rangle$ and $\left|1_{\mathrm{L}}\right\rangle$ are the logical zero and one states. Applying the AQEC quantum operation $\mathcal{P}+\mathcal{R} \circ \mathcal{P}_{\perp}$ infinitely fast, the effective evolution would be (up to the first order of $d t[31,33])$

$$
\begin{aligned}
\frac{d \rho}{d t}= & -i[\omega \mathcal{P}(H), \rho]+\sum_{i=1}^{r}\left[\mathcal{P}\left(L_{i} \rho L_{i}^{\dagger}\right)\right. \\
& \left.+\mathcal{R}\left(\mathcal{P}_{\perp}\left(L_{i} \rho L_{i}^{\dagger}\right)\right)-\frac{1}{2}\left\{\mathcal{P}\left(L_{i}^{\dagger} L_{i}\right), \rho\right\}\right],
\end{aligned}
$$


where $P_{\perp}=1-P, \mathcal{P}(\cdot)=P(\cdot) P, \mathcal{P}_{\perp}(\cdot)=P_{\perp}(\cdot) P_{\perp}$ and $\mathcal{R}$ is a CPTP map describing the AQEC recovery channel. We define the following class of AQEC codes:

$$
\left|0_{\mathrm{L}} / 1_{\mathrm{L}}\right\rangle=\sum_{i j} A_{0 / 1, i j}|i\rangle_{\mathcal{H}_{S}}|j, 0 / 1\rangle_{\mathcal{H}_{A}}
$$

where $A_{0}, A_{1} \in \mathbb{C}^{d \times d}, A_{0, i j}=C_{i j}+\varepsilon D_{i j}$, and $A_{1, i j}=C_{i j}-$ $\varepsilon D_{i j}$ satisfy $\operatorname{Tr}\left(A_{0} A_{0}^{\dagger}\right)=\operatorname{Tr}\left(A_{1} A_{1}^{\dagger}\right)=1$ and $\operatorname{Tr}\left(C^{\dagger} D\right)=0$. Here, $C$ describes the part of the code which $\left|0_{\mathrm{L}}\right\rangle$ and $\left|1_{\mathrm{L}}\right\rangle$ have in common and $D$ describes the part distinguishing $\left|0_{\mathrm{L}}\right\rangle$ from $\left|1_{L}\right\rangle$ which generates nonzero signal and noise. In the special case where $\varepsilon=0$, the effective signal and noise are zero. Let $\mathcal{H}_{A}=\mathcal{H}_{A^{\prime}} \otimes \mathcal{H}_{2}$, where $\operatorname{dim} \mathcal{H}_{A^{\prime}}=d$ and $\operatorname{dim} \mathcal{H}_{2}=2$, and the last ancillary qubit in $\mathcal{H}_{2}$ makes both the signal and noises diagonal in the code space, i.e., $\left\langle 0_{\mathrm{L}}|H| 1_{\mathrm{L}}\right\rangle=\left\langle 0_{\mathrm{L}}|S| 1_{\mathrm{L}}\right\rangle=0$ for all $S \in \mathcal{S}$. Later on, we will assume $\varepsilon$ is a small parameter and consider the perturbation expansion of the effective dynamics around $\varepsilon=0$. We consider the recovery channel restricted to the structure (we will show that this type of recovery channels is sufficient for our purpose),

$$
\begin{array}{r}
\mathcal{R}(\cdot)=\sum_{m}\left(\left|0_{\mathrm{L}}\right\rangle\left\langle R_{m}, 0|+| 1_{\mathrm{L}}\right\rangle\left\langle S_{m}, 1\right|\right) \\
(\cdot)\left(\left|R_{m}, 0\right\rangle\left\langle 0_{\mathrm{L}}|+| S_{m}, 1\right\rangle\left\langle 1_{\mathrm{L}}\right|\right),
\end{array}
$$

where $\left\{\left|R_{m}\right\rangle\right\},\left\{\left|S_{m}\right\rangle\right\} \subset \mathcal{H}_{S} \otimes \mathcal{H}_{A^{\prime}}$ are two sets of orthonormal basis and $\mathcal{R}$ is CPTP. A few lines of the calculation show the effective channel [Eq. (6)] under the AQEC code [Eq. (7)], and the recovery channel [Eq. (8)] is

$$
\frac{d \rho}{d t}=-i\left[\frac{\omega \operatorname{Tr}\left(H Z_{\mathrm{L}}\right)}{2} Z_{\mathrm{L}}+H_{\mathrm{S}}, \rho\right]+\frac{\gamma(\mathcal{R})}{2}\left(Z_{\mathrm{L}} \rho Z_{\mathrm{L}}-\rho\right),
$$

where $Z_{\mathrm{L}}=\left|0_{\mathrm{L}}\right\rangle\left\langle 0_{\mathrm{L}}|-| 1_{\mathrm{L}}\right\rangle\left\langle 1_{\mathrm{L}}\right|, H_{\mathrm{S}}$ is independent of $\omega$, and

$$
\begin{aligned}
\gamma(\mathcal{R})= & -\operatorname{Re}\left[\sum _ { i = 1 } ^ { r } \langle 0 _ { \mathrm { L } } | \left(\mathcal{R}\left(\mathcal{P}_{\perp}\left(L_{i}\left|0_{\mathrm{L}}\right\rangle\left\langle 1_{\mathrm{L}}\right| L_{i}^{\dagger}\right)\right)\right.\right. \\
& \left.\left.+\mathcal{P}\left(L_{i}\left|0_{\mathrm{L}}\right\rangle\left\langle 1_{\mathrm{L}}\right| L_{i}^{\dagger}\right)-\frac{1}{2}\left\{\mathcal{P}\left(L_{i}^{\dagger} L_{i}\right),\left|0_{\mathrm{L}}\right\rangle\left\langle 1_{\mathrm{L}}\right|\right\}\right)\left|1_{\mathrm{L}}\right\rangle\right] .
\end{aligned}
$$

We can remove the term $H_{\mathrm{S}}$ in Eq. (9) by applying a reverse Hamiltonian constantly [29]. For dephasing channels, the optimal $\mathfrak{F}$ is reached using a special type of spin-squeezed state as the input $[9-11,39,42]$, where we have

$$
\mathfrak{F}=\frac{\operatorname{Tr}\left(H Z_{\mathrm{L}}\right)^{2}}{2 \gamma(\mathcal{R})} \text {. }
$$

To simulate the evolution of multipartite spin-squeezed states using the sequential strategy where we have only a single probe, one could first prepare the desired spin-squeezed state in $\bigotimes_{i=1}^{N} \mathcal{H}_{i}$ by entangling the logical qubit in the effective dephasing channel $\left(\mathcal{H}_{1}=\mathcal{H}_{S} \otimes \mathcal{H}_{A}\right)$ with a large number of ancillas $\left(\bigotimes_{i=2}^{N} \mathcal{H}_{i}\right)$, where $\operatorname{dim} \mathcal{H}_{i}=\operatorname{dim} \mathcal{H}_{1}$ for $2 \leqslant i \leqslant N$, and then perform swap operations between $\mathcal{H}_{1}$ and $\mathcal{H}_{i}$ for $i=2, \ldots, N$ successively every time $t / N$. The optimal $\mathfrak{F}$ in Eq. (11) is asymptotically attainable at $N \rightarrow \infty$ [11]. On the other hand, if we used a single logical qubit state $\left|+_{L}\right\rangle=$ $\frac{\left|0_{\mathrm{L}}\right\rangle+\left|1_{\mathrm{L}}\right\rangle}{\sqrt{2}}$ as the input, the normalized QFI will be reduced by a factor of $e$, in which case one can still achieve $\mathfrak{F} \approx$ $\frac{4}{e} \min _{h, \mathbf{h}, \mathfrak{h} \mid \beta=0}\|\alpha\|$ for arbitrary Markovian noise.

For simplicity in future calculations, we perform a two-step gauge transformation on the Lindblad operators $\left\{L_{i}\right\}_{i=1}^{r}$ to simplify the dynamics: (1) Let $L_{i} \leftarrow L_{i}-\operatorname{Tr}\left(C^{\dagger} L_{i} C\right) \cdot \mathbb{1}$, such that $L_{i}$ satisfies $\operatorname{Tr}\left(C^{\dagger} L_{i} C\right)=0$ for all $L_{i}$. (2) Perform a unitary transformation $\mathbf{L} \leftarrow u \mathbf{L}\left(u \in \mathbb{C}^{r \times r}\right)$ such that $\operatorname{Tr}\left(C^{\dagger} L_{i}^{\dagger} L_{j} C\right)$ is a diagonal matrix. The transformations above only induce another parameter-independent shift $H_{\mathrm{S}}$ in the Hamiltonian, which could be eliminated by applying a reverse Hamiltonian. Now we have a new set of Lindblad operators $\left\{J_{i}\right\}_{i=1}^{r}$, satisfying

$$
\operatorname{Tr}\left(C^{\dagger} J_{i} C\right)=0, \quad \operatorname{Tr}\left(C^{\dagger} J_{i}^{\dagger} J_{j} C\right)=\lambda_{i} \delta_{i j},
$$

and we replace $\left\{L_{i}\right\}_{i=1}^{r}$ with $\left\{J_{i}\right\}_{i=1}^{r}$ in Eq. (10).

First, we maximize $\mathfrak{F}$ over the recovery $\mathcal{R}$, which is equivalent to minimizing $\gamma(\mathcal{R})$ over $\mathcal{R}$. We claim that the minimum noise rate $\gamma=\min _{\mathcal{R}} \gamma(\mathcal{R})$ is

$$
\begin{aligned}
\gamma= & -\left\|\sum_{i=1}^{r} \mathcal{P}_{\perp}\left(J_{i}\left|0_{\mathrm{L}}\right\rangle\left\langle 1_{\mathrm{L}}\right| J_{i}^{\dagger}\right)\right\|_{1}-\operatorname{Re}\left[\sum_{i=1}^{r}\left\langle 0_{\mathrm{L}}\right|\right. \\
& \left.\left(\mathcal{P}\left(J_{i}\left|0_{\mathrm{L}}\right\rangle\left\langle 1_{\mathrm{L}}\right| J_{i}^{\dagger}\right)-\frac{1}{2}\left\{\mathcal{P}\left(J_{i}^{\dagger} J_{i}\right),\left|0_{\mathrm{L}}\right\rangle\left\langle 1_{\mathrm{L}}\right|\right\}\right)\left|1_{\mathrm{L}}\right\rangle\right],
\end{aligned}
$$

where we have used $\max _{U: U^{\dagger} U=\mathbb{1}} \operatorname{Tr}\left(M U+M^{\dagger} U^{\dagger}\right)=2\|M\|_{1}$ for arbitrary square matrices $M$ and $U$, where $\|\cdot\|_{1}$ is the trace norm (see details in Appendix A).

Next, we would like to maximize $\mathfrak{F}$ [Eq. (11)] over all possible AQEC codes [Eq. (7)], which is mathematically difficult because of the trace norm in the denominator. To eliminate the trace norm, we further sacrifice the generality of our AQEC code and assume $\varepsilon \ll 1$. We call it the "perturbation" code in the sense that the signal and the noise are both infinitesimally small when $\varepsilon \rightarrow 0$. Under the limit $\varepsilon \rightarrow 0$, we have $\operatorname{Tr}\left(H Z_{\mathrm{L}}\right)=2 \varepsilon \operatorname{Tr}(H \tilde{C})+O\left(\varepsilon^{2}\right)$, where

$$
\tilde{C}=C D^{\dagger}+D C^{\dagger},
$$

and ignoring all $o\left(\varepsilon^{2}\right)$ terms [where $f(\varepsilon)=o\left(\varepsilon^{2}\right)$ means $\left.\lim _{\varepsilon \rightarrow 0} f(\varepsilon) / \varepsilon^{2}=0\right]$, the noise rate is

$$
\gamma=\varepsilon^{2}\left(\sum_{i} 2\left|\operatorname{Tr}\left(J_{i} \tilde{C}\right)\right|^{2}+\sum_{i j: \lambda_{i}+\lambda_{j} \neq 0} \frac{\left|\operatorname{Tr}\left(J_{i}^{\dagger} J_{j} \tilde{C}\right)\right|^{2}}{\left(\lambda_{i}+\lambda_{j}\right)}\right) .
$$

For a detailed derivation of the noise rate, see Appendix B and [63]. Finally, we have the following expression of the normalized QFI (up to the lowest order of $\varepsilon$ ):

$$
\mathfrak{F}(C, \tilde{C}) \approx \frac{\operatorname{Tr}(H \tilde{C})^{2}}{\sum_{i}\left|\operatorname{Tr}\left(J_{i} \tilde{C}\right)\right|^{2}+\sum_{i j: \lambda_{i}+\lambda_{j} \neq 0} \frac{\left|\operatorname{Tr}\left(J_{i}^{\dagger} J_{j} \tilde{C}\right)\right|^{2}}{2\left(\lambda_{i}+\lambda_{j}\right)},}
$$

as a function of $\tilde{C}$ and $C$ (implicitly through the choice of $\left.\left\{J_{i}\right\}_{i=1}^{r}\right)$. The effective dynamics of the perturbation code has the feature that both the signal and the noises are equally weak and only the ratio between them matters. Therefore, the exact value of $\varepsilon$ will not influence the normalized QFI $\mathfrak{F}$ as long as it is sufficiently small. On the other hand, it does influence how fast $F(t) / t$ reaches its optimum $\mathfrak{F}$, characterized by a coherence time $O\left(1 / \varepsilon^{2}\right)$. 


\section{SATURATING THE BOUND}

Now we maximize the normalized QFI (up to the lowest order of $\varepsilon$ ) over $C$ and $\tilde{C}$ and show that the optimal $\mathfrak{F}$ is exactly equal to its upper bound in Eq. (2). The domain of $C$ is all complex matrices satisfying $\operatorname{Tr}\left(C^{\dagger} C\right)=1$. We assume the domain of $\tilde{C}$ is all traceless Hermitian matrices satisfying $\operatorname{Tr}\left(J_{i}^{\dagger} J_{j} \tilde{C}\right)=0$ for all $i, j \in \mathfrak{n}:=\left\{i \mid \lambda_{i}=0\right\}$. When $C$ is full rank, $\mathfrak{n}$ is empty, and for arbitrary traceless $\tilde{C}$, we could always take $D^{\dagger}=\frac{1}{2} C^{-1} \tilde{C}$ such that Eq. (14) is satisfied. When $C$ is singular, we could replace it with an approximate full-rank version (e.g., $C \leftarrow C+\delta \mathbb{1}$ ). In this case, $\mathfrak{F}$ will only be decreased by an infinitesimally small amount when $\varepsilon=o\left(\delta^{2}\right)$ because the numerator in Eq. (16) is only slightly perturbed after the replacement.

Consider the following optimization problem over $h, \mathbf{h}, \mathfrak{h}$, and $C$ :

$$
\begin{aligned}
& \max _{C} \min _{h, \mathbf{h}, \mathfrak{h}} 4 \operatorname{Tr}\left(C^{\dagger} \alpha C\right), \\
& \text { subject to } \beta=0, \quad \operatorname{Tr}\left(C^{\dagger} C\right)=1 .
\end{aligned}
$$

Fixing $C$, we introduce a Hermitian matrix $\tilde{C}$ as the Lagrange multiplier associated with the constraint $\beta=0$ [64]. Strong duality implies Eq. (17) has the same solution as the following dual program (see Appendix C):

$$
\begin{gathered}
\max _{C, \tilde{C}} \mathfrak{F}(C, \tilde{C}), \quad \text { subject to } \operatorname{Tr}\left(C^{\dagger} C\right)=1, \quad \operatorname{Tr}(\tilde{C})=0, \\
\text { and } \operatorname{Tr}\left(J_{i}^{\dagger} J_{j} \tilde{C}\right)=0, \quad \forall i, j \in \mathfrak{n},
\end{gathered}
$$

whose optimal value could be achieved using the perturbation code up to an infinitesimally small error according to the discussion above. On the other hand, thanks to Sion's minimax theorem $[65,66]$, we can exchange the order of the maximization and minimization in Eq. (17) (see Appendix D). Therefore, the optimal value of Eq. (18) is also equal to $4 \min _{h, \mathbf{h}, \mathfrak{h} \mid \beta=0}\|\alpha\|$, the upper bound of the normalized QFI.

\section{NUMERICAL ALGORITHM}

It is known that the upper bound in Eq. (2) could be calculated via a semidefinite program (SDP) $[30,44]$,

$$
F(t) \leqslant 4 t \min _{h, \mathbf{h}, \mathfrak{h}} x, \quad \text { subject to } \mathscr{A} \succeq 0, \quad \beta=0,
$$

where $\mathscr{A}=\left(\begin{array}{cc}\sqrt{x} \mathbb{1} & \mathbf{h}^{\dagger} \mathbb{1}+\mathbf{L}^{\dagger} \mathfrak{h} \mathfrak{h}+\mathfrak{h} \mathbf{L} \\ \sqrt{x} \mathbb{1}^{\otimes^{\prime}}\end{array}\right)$ and $\succeq 0$ means positive semidefinite. However, the minimax theorem does not guarantee an efficient algorithm to solve Eq. (18) after exchanging the order of the maximization and minimization in Eq. (17). Now we provide an efficient numerical algorithm, obtaining an optimal $\left(C^{\diamond}, \tilde{C}^{\diamond}\right)$ in three steps. The validity of this algorithm is proven in Appendix E. The algorithm runs as follows: (a) Solving $\min _{h, \mathbf{h}, \mathfrak{h} \mid \beta=0}\|\alpha\|$ using the SDP gives us an optimal $\alpha^{\diamond}$ (and corresponding $h^{\diamond}, \mathbf{h}^{\diamond}, \mathfrak{h}^{\diamond}$ ) satisfying $\left\|\alpha^{\diamond}\right\|=\min _{h, \mathbf{h}, \mathfrak{h} \mid \beta=0}\|\alpha\|$. (b) Suppose $\Pi^{\diamond}$ is the projection onto the subspace spanned by all eigenstates corresponding to the largest eigenvalue of $\alpha^{\diamond}$, we find an optimal $C^{\diamond} C^{\diamond \dagger}$ satisfying $\Pi^{\diamond} C^{\diamond} C^{\diamond \dagger} \Pi^{\diamond}=C^{\diamond} C^{\diamond \dagger}$ and

$$
\operatorname{Re}\left\{\operatorname{Tr}\left[C^{\diamond} C^{\diamond \dagger}(\Delta \mathbf{h} \mathbb{1}+\Delta \mathfrak{h} \mathbf{L})^{\dagger}\left(\mathbf{h}^{\diamond} \mathbb{1}+\mathfrak{h}^{\diamond} \mathbf{L}\right)\right]\right\}=0,
$$

for all $(\Delta \mathbf{h}, \Delta \mathfrak{h})$ such that $\Delta h \mathbb{1}+\Delta \mathbf{h}^{\dagger} \mathbf{L}+\mathbf{L}^{\dagger} \Delta \mathbf{h}+$ $\mathbf{L}^{\dagger} \Delta \mathfrak{h} \mathbf{L}=0$ for some $\Delta h$. Note that this step is simply solving a system of linear equations. (c) Find $\left\{J_{i}\right\}_{i=1}^{r}$ via the gauge transformation. Let $\mathcal{S}_{0}=\operatorname{span}\left\{I, J_{i}^{\dagger} J_{j}, \forall i, j \in \mathfrak{n}\right\}$. Decompose $M=J_{i}$ or $J_{i j}\left(:=J_{i}^{\dagger} J_{j}\right)$ into $M=M^{h}+i M^{a h}+$ $M_{0}^{h}+i M_{0}^{a h}$, where $M^{h, a h}, M_{0}^{h, a h}$ are Hermitian, $M_{0}^{h, a h} \in \mathcal{S}_{0}$ and $M^{h, a h} \perp \mathcal{S}_{0}$ (in terms of the Hilbert-Schmidt norm). Using the vectorization of matrices $|\cdot\rangle\rangle=\sum_{j k}\langle j|(\cdot)| k\rangle|j\rangle|k\rangle$, let

$$
\begin{aligned}
B= & \left.\sum_{i}\left|J_{i}^{h}\right\rangle\right\rangle\left\langle J_{i}^{h}|+| J_{i}^{a h}\right\rangle\left\langle\left\langle\left\langle J_{i}^{a h}\right|\right.\right. \\
& +\sum_{i j: \lambda_{i}+\lambda_{j} \neq 0} \frac{\left.\left|J_{i j}^{h}\right\rangle\right\rangle\left\langle\langle J _ { i j } ^ { h } | + | J _ { i j } ^ { a h } \rangle \left\langle\left\langle\left\langle J_{i j}^{a h}\right|\right.\right.\right.}{2\left(\lambda_{i}+\lambda_{j}\right)} .
\end{aligned}
$$

According to the Cauchy-Schwarz inequality,

$$
\max _{\tilde{C}} \mathfrak{F}\left(C^{\diamond}, \tilde{C}\right)=\max _{\tilde{C}} \frac{\left.|\langle H \mid \tilde{C}\rangle\rangle\right|^{2}}{\langle\langle\tilde{C}|B| \tilde{C}\rangle\rangle}=\left\langle\left\langle H^{h}\left|B^{-1}\right| H^{h}\right\rangle\right\rangle,
$$

and the optimal $\left.\left.\left|\tilde{C}^{\diamond}\right\rangle\right\rangle=B^{-1}\left|H^{h}\right\rangle\right\rangle$. Here, the superscript ${ }^{-1}$ means the Moore-Penrose pseudoinverse.

\section{HIGHLY BIASED NOISE}

We consider a special case where noises are separated into two groups-strong ones and weak ones [31-33]. To be specific, we consider the following quantum master equation:

$$
\begin{aligned}
\frac{d \rho}{d t}= & -i[\omega H, \rho]+\sum_{i \in \overline{\mathfrak{s}}} \eta\left(L_{i} \rho L_{i}^{\dagger}-\frac{1}{2}\left\{L_{i}^{\dagger} L_{i}, \rho\right\}\right) \\
& +\sum_{i \in \mathfrak{s}}\left(L_{i} \rho L_{i}^{\dagger}-\frac{1}{2}\left\{L_{i}^{\dagger} L_{i}, \rho\right\}\right),
\end{aligned}
$$

where the indices of the Lindblad operators $\left\{L_{i}\right\}_{i=1}^{r}$ are separated into $\overline{\mathfrak{s}}$ and $\mathfrak{s}$, representing weak and strong noises respectively. $\eta \ll 1$ is a small parameter characterizing the relative strength of the weak noises. Moreover, we assume that $H \notin \operatorname{span}\left\{\mathbb{1}, L_{i}, L_{i}^{\dagger}, L_{i}^{\dagger} L_{j}, i, j \in \mathfrak{s}\right\}$ so that it is possible to fully correct all strong noises and also preserve a nontrivial signal in the code space. Taking $\eta \rightarrow 0$, we can show that (see Appendix F) the asymptotically attainable upper bound $4 \min _{h, \mathbf{h}, \mathfrak{h} \mid \beta=0}\|\alpha\|$ is equal to

$$
\mathfrak{F}^{\diamond}=\frac{1}{\eta}\|\bar{\alpha}\|_{h, \mathbf{h}, \mathfrak{h} \mid \beta=0}+O(1),
$$

where $\bar{\alpha}=(\mathbf{h} \mathbb{1}+\mathfrak{h} \mathbf{L})^{\dagger} \Pi_{\overline{\mathfrak{s}}}(\mathbf{h} \mathbb{1}+\mathfrak{h} \mathbf{L})$ and $\Pi_{\overline{\mathfrak{s}}}$ is a diagonal matrix whose $i$ th diagonal element is one when $i \in \overline{\mathfrak{s}}$, and zero when $i \in \mathfrak{s}$. This reduces the running time of the SDP in Eq. (19) by reducing $\mathscr{A}$ from a $d(r+1) \times d(r+1)$ matrix to a $d(|\overline{\mathfrak{s}}|+1) \times d(|\overline{\mathfrak{s}}|+1)$ matrix. Using the optimal AQEC strategy, $\mathfrak{F}$ is boosted by a factor of $O(1 / \eta)$, compared to the case where no QEC is performed. To find the optimal AQEC code, we can solve the dual program of a modified version of Eq. (17) where $\alpha$ is replaced with $\bar{\alpha}$ :

$$
\begin{gathered}
\max _{C, \tilde{C}} \overline{\mathfrak{F}}(C, \tilde{C}), \quad \text { subject to } \operatorname{Tr}\left(C^{\dagger} C\right)=1, \quad \operatorname{Tr}(\tilde{C})=0, \\
\forall_{i, j \in \mathfrak{s}}, \operatorname{Tr}\left(L_{i}^{\dagger} L_{j} \tilde{C}\right)=\operatorname{Tr}\left(L_{i} \tilde{C}\right)=0,
\end{gathered}
$$


and some other linear constraints on $\tilde{C}$ when $\operatorname{Tr}\left(C \mathbf{L}^{\dagger} \mathbf{L} C\right)-$ $\operatorname{Tr}\left(C \mathbf{L}^{\dagger} C\right) \operatorname{Tr}(C \mathbf{L} C)$ is singular. Here, $\overline{\mathfrak{F}}$ is the dominant part of $\mathfrak{F}$ such that $\mathfrak{F}=\overline{\mathfrak{F}} / \eta+O(1)$. Details are provided in Appendix F. The constraint given by Eq. (26) on $\tilde{C}$ is equivalent to the Knill-Laflamme condition for Lindblad operators $\left\{L_{i}\right\}_{i \in \mathfrak{s}}[67,68]$. It implies that strong noises are fully corrected by the optimal AQEC code and explains why the estimation precision depends only on the strength of weak noises in Eq. (24).

\section{CONCLUSIONS AND OUTLOOK}

In this paper, we proposed an AQEC strategy such that the optimal SQL in the Hamiltonian parameter estimation could be achieved asymptotically. An interesting question is whether the perturbation code could be turned into nonperturbative ones. We provide an example in Appendix G, where by modifying the ancilla-free QEC code (nonperturbation) proposed in Ref. [33], we show that the optimal $\mathfrak{F}$ could be achieved in the correlated dephasing noise model. However, it is unclear how to generalize the result to generic noise models. Another two interesting open questions are (1) how to characterize the power of QEC in improving quantum metrology for general quantum channels $[41,42]$, for example when the rate of quantum controls is constant, rather than infinitely fast; and (2) how to optimize the QEC strategy when considering a constant probing time, rather than an infinitely long probing time.

\section{ACKNOWLEDGMENTS}

We thank Kyungjoo Noh, Rafał Demkowicz-Dobrzański, Zhou Fan, Jing Yang, and Yuxiang Yang for helpful discussions. We acknowledge support from the ARL-CDQI (Grants No. W911NF15-2-0067 and No. W911NF-18-2-0237), ARO (Grants No. W911NF-18-1-0020 and No. W911NF-18-10212), ARO MURI (Grant No. W911NF-16-1-0349), AFOSR MURI (Grant No. FA9550-15-1-0015), U.S. Department of Energy (Grant No. DE-SC0019406), NSF (Grant No. EFMA1640959), and the Packard Foundation (Grant No. 201339273).

\section{APPENDIX A: MINIMIZING THE NOISE RATE $\boldsymbol{\gamma}(\mathcal{R})$ OVER RECOVERY CHANNELS $\mathcal{R}$}

In this Appendix, we prove Eq. (13) in the main text. According to Eq. (10),

$$
\gamma(\mathcal{R})=-\operatorname{Re}\left\{\sum_{i=1}^{r}\left\langle 0_{\mathrm{L}}\left|\left[\mathcal{R}\left(\mathcal{P}_{\perp}\left(J_{i}\left|0_{\mathrm{L}}\right\rangle\left\langle 1_{\mathrm{L}}\right| J_{i}^{\dagger}\right)\right)+\mathcal{P}\left(J_{i}\left|0_{\mathrm{L}}\right\rangle\left\langle 1_{\mathrm{L}}\right| J_{i}^{\dagger}\right)-\frac{1}{2}\left\{\mathcal{P}\left(J_{i}^{\dagger} J_{i}\right),\left|0_{\mathrm{L}}\right\rangle\left\langle 1_{\mathrm{L}}\right|\right\}\right]\right| 1_{\mathrm{L}}\right\rangle\right\} .
$$

In order to calculate $\gamma=\min _{\mathcal{R}} \gamma(\mathcal{R})$, we only need to calculate the first term minimized over $\mathcal{R}$ :

$$
\begin{aligned}
-\max _{\mathcal{R}} \operatorname{Re}\left[\sum_{i}\left\langle 0_{\mathrm{L}}\left|\mathcal{R}\left(\mathcal{P}_{\perp}\left(J_{i}\left|0_{\mathrm{L}}\right\rangle\left\langle 1_{\mathrm{L}}\right| J_{i}^{\dagger}\right)\right)\right| 1_{\mathrm{L}}\right\rangle\right] & =-\max _{\left|R_{m}\right\rangle,\left|S_{m}\right\rangle} \operatorname{Re}\left[\sum_{i, m}\left\langle R_{m}, 0\left|P_{\perp} J_{i}\right| 0_{\mathrm{L}}\right\rangle\left\langle 1_{\mathrm{L}}\left|J_{i}^{\dagger} P_{\perp}\right| S_{m}, 1\right\rangle\right] \\
& =-\frac{1}{2} \max _{\left|R_{m}\right\rangle,\left|S_{m}\right\rangle} \operatorname{Tr}\left(\sum_{m}\left|R_{m}\right\rangle\left\langle S_{m}\right| \cdot \sum_{i}\left\langle 0\left|P_{\perp} J_{i}\right| 0_{\mathrm{L}}\right\rangle\left\langle 1_{\mathrm{L}}\left|J_{i}^{\dagger} P_{\perp}\right| 1\right\rangle+\mathrm{H} . \mathrm{c} .\right) \\
& =-\left\|\sum_{i}\left\langle 0\left|P_{\perp} J_{i}\right| 0_{\mathrm{L}}\right\rangle\left\langle 1_{\mathrm{L}}\left|J_{i}^{\dagger} P_{\perp}\right| 1\right\rangle\right\|_{1}=-\| \sum_{i} P_{\perp} J_{i}\left|0_{\mathrm{L}}\right\rangle\left\langle 1_{\mathrm{L}}\right| J_{i}^{\dagger} P_{\perp}\|\|_{1}^{\prime}
\end{aligned}
$$

where H.c. means Hermitian conjugate and we have used $\max _{U: U^{\dagger} U=\mathbb{1}} \operatorname{Tr}\left(M U+M^{\dagger} U^{\dagger}\right)=2\|M\|_{1}$ for arbitrary square matrices $M$ and $U$, which could be easily proven using the singular-value decomposition of $M$.

\section{APPENDIX B: PERTURBATION EXPANSION OF THE NOISE RATE $\gamma$}

In this Appendix, we expand the minimum noise rate $\gamma$ around $\varepsilon=0$ using the perturbation code. For simplicity, the equal sign "=" in this Appendix means approximate equality up to the second order of $\varepsilon$ [ignoring all $o\left(\varepsilon^{2}\right)$ terms]. We also state a useful lemma here:

Lemma 1 [69]. $\|X+\varepsilon Y\|_{1}=\|X\|_{1}+O(\varepsilon)$ for arbitrary $X$ and $Y$.

To calculate Eq. (13), we first consider the terms independent of $\mathcal{R}$,

$$
\begin{aligned}
-\operatorname{Re}\left[\sum_{i=1}^{r}\left\langle 0_{\mathrm{L}}\left|\left(\mathcal{P}\left(J_{i}\left|0_{\mathrm{L}}\right\rangle\left\langle 1_{\mathrm{L}}\right| J_{i}^{\dagger}\right)-\frac{1}{2}\left\{\mathcal{P}\left(J_{i}^{\dagger} J_{i}\right),\left|0_{\mathrm{L}}\right\rangle\left\langle 1_{\mathrm{L}}\right|\right\}\right)\right| 1_{\mathrm{L}}\right\rangle\right] \\
=-\sum_{i} \operatorname{Re}\left[\operatorname{Tr}\left(A_{0} A_{0}^{\dagger} J_{i}\right) \operatorname{Tr}\left(A_{1} A_{1}^{\dagger} J_{i}^{\dagger}\right)\right]-\frac{1}{2}\left[\operatorname{Tr}\left(A_{0} A_{0}^{\dagger} J_{i}^{\dagger} J_{i}\right)+\operatorname{Tr}\left(A_{1} A_{1}^{\dagger} J_{i}^{\dagger} J_{i}\right)\right] \\
=\sum_{i} \lambda_{i}+\varepsilon^{2}\left|\operatorname{Tr}\left(\tilde{C} J_{i}\right)\right|^{2}+\varepsilon^{2} \operatorname{Tr}\left(D D^{\dagger} J_{i}^{\dagger} J_{i}\right) .
\end{aligned}
$$


The remaining term is equal to (thanks to Lemma 1) -1 times the following quantity:

$$
\| \sum_{i} P_{\perp} J_{i}\left|0_{\mathrm{L}}\right\rangle\left\langle 1_{\mathrm{L}}\right| J_{i}^{\dagger} P_{\perp}\left\|_{1}=\right\|\left(\begin{array}{c}
\sqrt{\tilde{\Lambda}^{-1}}\left(\Lambda+\varepsilon X_{1}+\varepsilon^{2} X_{1}^{\prime}\right) \\
\varepsilon X_{2}+\varepsilon^{2} X_{2}^{\prime}
\end{array}\right)\left[\left(\Lambda-\varepsilon X_{1}+\varepsilon^{2} X_{1}^{\prime}\right)^{\dagger} \sqrt{\tilde{\Lambda}^{-1}}-\varepsilon X_{2}^{\dagger}+\varepsilon^{2} X_{2}^{\prime \dagger}\right] \|_{1},
$$

where $\Lambda \in \mathbb{R}^{r \times r}$ is a diagonal matrix whose $k$ th diagonal element is $\lambda_{k}$, and $\tilde{\Lambda} \in \mathbb{R}^{r \times r}$ is a diagonal matrix whose $k$ th diagonal element is $\lambda_{k}$ if $\lambda_{k}>0$, and 1 if $\lambda_{k}=0$. Assume $\left\{\lambda_{k}\right\}_{k=1}^{r}$ is arranged in a nonascending order and $r_{0}$ is the largest integer such that $\lambda_{r_{0}}$ is positive. $X_{1}, X_{1}^{\prime} \in \mathbb{C}^{r \times r}$ satisfy

$$
\begin{aligned}
\left(\Lambda+\varepsilon X_{1}+\varepsilon^{2} X_{1}^{\prime}\right)_{j i} & =\sqrt{\lambda_{j}}\left\langle\tilde{J}_{j, 0}\left|P_{\perp} J_{i}\right| 0_{\mathrm{L}}\right\rangle=\operatorname{Tr}\left(C^{\dagger} J_{j}^{\dagger} J_{i} A_{0}\right)-\operatorname{Tr}\left(C^{\dagger} J_{j}^{\dagger} A_{0}\right) \operatorname{Tr}\left(A_{0}^{\dagger} J_{i} A_{0}\right) \\
& =\lambda_{i} \delta_{i j}+\varepsilon \operatorname{Tr}\left(C^{\dagger} J_{j}^{\dagger} J_{i} D\right)-\varepsilon^{2} \operatorname{Tr}\left(C^{\dagger} J_{j}^{\dagger} D\right) \operatorname{Tr}\left(\tilde{C} J_{i}\right),
\end{aligned}
$$

for $1 \leqslant j \leqslant r_{0}$, and

$$
\begin{aligned}
\left(\Lambda+\varepsilon X_{1}+\varepsilon^{2} X_{1}^{\prime}\right)_{j i} & =\left\langle\tilde{J}_{j, 0}\left|P_{\perp} J_{i}\right| 0_{\mathrm{L}}\right\rangle=\operatorname{Tr}\left(\tilde{J}_{j}^{\dagger} J_{i} A_{0}\right)-\operatorname{Tr}\left(\tilde{J}_{j}^{\dagger} A_{0}\right) \operatorname{Tr}\left(A_{0}^{\dagger} J_{i} A_{0}\right) \\
& =\varepsilon \operatorname{Tr}\left(\tilde{J}_{j}^{\dagger} J_{i} D\right)-\varepsilon^{2} \operatorname{Tr}\left(\tilde{J}_{j}^{\dagger} D\right) \operatorname{Tr}\left(\tilde{C} J_{i}\right),
\end{aligned}
$$

for $r_{0}+1 \leqslant j \leqslant r . X_{2}, X_{2}^{\prime} \in \mathbb{C}^{\left(d^{2}-r\right) \times r}$ satisfy

$$
\begin{aligned}
\left(\Lambda+\varepsilon X_{2}+\varepsilon^{2} X_{2}^{\prime}\right)_{(j-r) i} & =\left\langle\tilde{J}_{j, 0}\left|P_{\perp} J_{i}\right| 0_{\mathrm{L}}\right\rangle=\operatorname{Tr}\left(\tilde{J}_{j}^{\dagger} J_{i} A_{0}\right)-\operatorname{Tr}\left(\tilde{J}_{j}^{\dagger} A_{0}\right) \operatorname{Tr}\left(A_{0}^{\dagger} J_{i} A_{0}\right) \\
& =\varepsilon \operatorname{Tr}\left(\tilde{J}_{j}^{\dagger} J_{i} D\right)-\varepsilon^{2} \operatorname{Tr}\left(\tilde{J}_{j}^{\dagger} D\right) \operatorname{Tr}\left(\tilde{C} J_{i}\right)
\end{aligned}
$$

for $r+1 \leqslant j \leqslant d^{2}-1$, and

$$
\begin{aligned}
\left(\Lambda+\varepsilon X_{2}+\varepsilon^{2} X_{2}^{\prime}\right)_{\left(d^{2}-r\right) i} & =\left\langle\tilde{J}_{d^{2}, 0}\left|P_{\perp} J_{i}\right| 0_{\mathrm{L}}\right\rangle=\operatorname{Tr}\left(\tilde{J}_{d^{2}}^{\dagger} J_{i} A_{0}\right)-\operatorname{Tr}\left(\tilde{J}_{d^{2}}^{\dagger} A_{0}\right) \operatorname{Tr}\left(A_{0}^{\dagger} J_{i} A_{0}\right) \\
& =\varepsilon \operatorname{Tr}\left(C^{\dagger} J_{i} D\right)-\varepsilon \operatorname{Tr}\left(\tilde{C} J_{i}\right) .
\end{aligned}
$$

Here,

$$
\left|\tilde{J}_{j, 0 / 1}\right\rangle=\left\{\begin{array}{lc}
\frac{1}{\sqrt{\lambda_{j}}} \sum_{i k} C_{i k} J_{j}|i\rangle|k, 0 / 1\rangle, & j \leqslant r_{0}, \\
\sum_{i k}\left(\tilde{J}_{j}\right)_{i k}|i\rangle|k, 0 / 1\rangle, & r_{0}<j \leqslant d^{2}, \\
\sum_{i k} \frac{C_{i k}}{\sqrt{\operatorname{Tr}\left(C^{\dagger} C\right)}}|i\rangle|k, 0 / 1\rangle, & j=d^{2},
\end{array}\right.
$$

are two sets of orthonormal basis of $\mathcal{H}_{S} \otimes \mathcal{H}_{A}$

To calculate the first- and second-order expansion of Eq. (B2), we consider the singular-value decompositions,

$$
\begin{aligned}
\left(\begin{array}{c}
\sqrt{\tilde{\Lambda}^{-1}}\left(\Lambda+\varepsilon X_{1}+\varepsilon^{2} X_{1}^{\prime}\right) \\
\varepsilon X_{2}+\varepsilon^{2} X_{2}^{\prime}
\end{array}\right) & =U(\varepsilon)\left(\begin{array}{c}
\Sigma(\varepsilon) \\
0
\end{array}\right) V(\varepsilon)^{\dagger}, \\
\left(\left(\Lambda-\varepsilon X_{1}+\varepsilon^{2} X_{1}^{\prime}\right)^{\dagger} \sqrt{\tilde{\Lambda}^{-1}}-\varepsilon X_{2}^{\dagger}+\varepsilon^{2} X_{2}^{\prime \dagger}\right) & =V(-\varepsilon)(\Sigma(-\varepsilon) \quad 0) U(-\varepsilon)^{\dagger} .
\end{aligned}
$$

Then,

$$
\begin{aligned}
\text { Eq. (B2) } & =\left\|U(\varepsilon)\left(\begin{array}{cc}
\Sigma(\varepsilon) V(\varepsilon)^{\dagger} V(-\varepsilon) \Sigma(-\varepsilon) & 0 \\
0 & 0
\end{array}\right) U(-\varepsilon)^{\dagger}\right\|_{1}=\left\|\Sigma(\varepsilon) V(\varepsilon)^{\dagger} V(-\varepsilon) \Sigma(-\varepsilon)\right\|_{1} \\
& =\operatorname{Tr}\left\{\left[V(\varepsilon) \Sigma(\varepsilon) V(\varepsilon)^{\dagger} V(-\varepsilon) \Sigma(-\varepsilon) \Sigma(-\varepsilon) V(-\varepsilon)^{\dagger} V(\varepsilon) \Sigma(\varepsilon) V(\varepsilon)^{\dagger}\right]^{1 / 2}\right\} \\
& =\operatorname{Tr}[\sqrt{\sqrt{Y(\varepsilon)} Y(-\varepsilon) \sqrt{Y(\varepsilon)}}],
\end{aligned}
$$

where

$$
\begin{aligned}
Y(\varepsilon)=V(\varepsilon) \Sigma(\varepsilon)^{2} V(\varepsilon)^{\dagger} & =\Lambda+\varepsilon\left(X_{1}^{\dagger} \Pi_{\Lambda}+\Pi_{\Lambda} X_{1}\right)+\varepsilon^{2}\left(X_{1}^{\dagger} \tilde{\Lambda}^{-1} X_{1}+X_{1}^{\prime} \Pi_{\Lambda}+\Pi_{\Lambda} X_{1}^{\prime \dagger}+X_{2}^{\dagger} X_{2}\right) \\
& =: \Lambda+\varepsilon W+\varepsilon^{2} W^{\prime},
\end{aligned}
$$

and $\Pi_{\Lambda}$ is the projector onto the support of $\Lambda$.

Using Theorem 2 in Ref. [63], we have

$$
\operatorname{Tr}[\sqrt{\sqrt{Y(\varepsilon)} Y(-\varepsilon) \sqrt{Y(\varepsilon)}}]=\operatorname{Tr}(\Lambda)+\varepsilon^{2} \operatorname{Tr}\left(X_{1}^{\dagger} \tilde{\Lambda}^{-1} X_{1}+X_{1}^{\prime} \Pi_{\Lambda}+\Pi_{\Lambda} X_{1}^{\prime \dagger}+X_{2}^{\dagger} X_{2}\right)-\varepsilon^{2} \sum_{i, j: \lambda_{i}+\lambda_{j} \neq 0}^{r} \frac{\left|X_{1, i j}+X_{1, j i}^{*}\right|^{2}}{\lambda_{i}+\lambda_{j}} .
$$


Note that

$$
\begin{array}{r}
\operatorname{Tr}\left(X_{1}^{\dagger} \tilde{\Lambda}^{-1} X_{1}+X_{2}^{\dagger} X_{2}\right)=\sum_{i=1}^{r} \operatorname{Tr}\left(D D^{\dagger} J_{i}^{\dagger} J_{i}\right)+\sum_{i=1}^{r}\left|\operatorname{Tr}\left(\tilde{C} J_{i}\right)\right|^{2}-\sum_{i=1}^{r} \operatorname{Tr}\left(D^{\dagger} J_{i}^{\dagger} C\right) \operatorname{Tr}\left(\tilde{C} J_{i}\right)+\operatorname{Tr}\left(C^{\dagger} J_{i} D\right) \operatorname{Tr}\left(\tilde{C} J_{i}^{\dagger}\right), \\
\operatorname{Tr}\left(X_{1}^{\prime} \Pi_{\Lambda}+\Pi_{\Lambda} X_{1}^{\prime \dagger}\right)=-\sum_{i=1}^{r}\left[\operatorname{Tr}\left(C^{\dagger} J_{i}^{\dagger} D\right) \operatorname{Tr}\left(\tilde{C} J_{i}\right)+\operatorname{Tr}\left(D^{\dagger} J_{i} C\right) \operatorname{Tr}\left(\tilde{C} J_{i}^{\dagger}\right)\right]
\end{array}
$$

and therefore

$$
\begin{aligned}
\gamma & =\text { Eq. (B1) - Eq. (B2) } \\
& =2 \varepsilon^{2} \sum_{i}\left|\operatorname{Tr}\left(J_{i} \tilde{C}\right)\right|^{2}+\varepsilon^{2} \sum_{i j: \lambda_{i}+\lambda_{j} \neq 0} \frac{\left|\operatorname{Tr}\left(J_{i}^{\dagger} J_{j} \tilde{C}\right)\right|^{2}}{\left(\lambda_{i}+\lambda_{j}\right)},
\end{aligned}
$$

where $\tilde{C}=C D^{\dagger}+D C^{\dagger}$. According to Eq. (11), we have

$$
\mathfrak{F}=\frac{\operatorname{Tr}(H \tilde{C})^{2}}{\sum_{i}\left|\operatorname{Tr}\left(J_{i} \tilde{C}\right)\right|^{2}+\sum_{i j: \lambda_{i}+\lambda_{j} \neq 0} \frac{\left|\operatorname{Tr}\left(J_{i}^{\dagger} J_{j} \tilde{C}\right)\right|^{2}}{2\left(\lambda_{i}+\lambda_{j}\right)}}+O(\varepsilon) .
$$

\section{APPENDIX C: LAGRANGE DUAL PROGRAM OF Eq. (17)}

Here we show that the Lagrange dual program of Eq. (17) is Eq. (18). From the definition of $\alpha$ [Eq. (3)] and $\beta$ [Eq. (4)], we see that the upper bound in Eq. (2) is invariant under the transformation $\mathbf{L} \rightarrow \mathbf{J}$; that is, after the transformation $\mathbf{L} \rightarrow \mathbf{J}$, there is always another set of $(h, \mathbf{h}, \mathfrak{h})$ such that $\beta=0$ and $\alpha$ is the same. Therefore, we let

$$
\begin{gathered}
\alpha=(\mathbf{h} \mathbb{1}+\mathfrak{h} \mathbf{J})^{\dagger}(\mathbf{h} \mathbb{1}+\mathfrak{h} \mathbf{J}), \\
\beta=H+h \mathbb{1}+\mathbf{h}^{\dagger} \mathbf{J}+\mathbf{J}^{\dagger} \mathbf{h}+\mathbf{J}^{\dagger} \mathfrak{h} \mathbf{J},
\end{gathered}
$$

where $\mathbf{J}=\left(J_{1}, J_{2}, \ldots, J_{r}\right)^{T}$. To proceed, we simplify the notations by letting

$$
j_{i}=\frac{\operatorname{Tr}\left(J_{i} \tilde{C}\right)}{\operatorname{Tr}(H \tilde{C})}, \quad j_{i j}=\frac{\operatorname{Tr}\left(J_{i}^{\dagger} J_{j} \tilde{C}\right)}{\operatorname{Tr}(H \tilde{C})} .
$$

Note that the $r$-dimensional vector $j$ is to be distinguished from the index $j$; then we have

$$
\mathfrak{F}(C, \tilde{C})=\left(j^{\dagger} j+\sum_{i j: \lambda_{i}+\lambda_{j} \neq 0} \frac{\left|\mathfrak{j}_{i j}\right|^{2}}{2\left(\lambda_{i}+\lambda_{j}\right)}\right)^{-1},
$$

and $4 \operatorname{Tr}\left(C^{\dagger} \alpha C\right)=4\left[\mathbf{h}^{\dagger} \mathbf{h}+\operatorname{Tr}\left(\Lambda \mathfrak{h}^{2}\right)\right]$.

Fixing $C$, we introduce a Hermitian matrix $\tilde{C}$ as a Lagrange multiplier of $\beta=0$ [64], and the Lagrange function is

$$
L(\tilde{C}, h, \mathbf{h}, \mathfrak{h})=4\left[\mathbf{h}^{\dagger} \mathbf{h}+\operatorname{Tr}\left(\Lambda \mathfrak{h}^{2}\right)\right]+\operatorname{Tr}\left[\tilde{C}\left(H+h \mathbb{1}+\mathbf{J}^{\dagger} \mathbf{h}+\mathbf{h}^{\dagger} \mathbf{J}+\mathbf{J}^{\dagger} \mathfrak{h} \mathbf{J}\right)\right]
$$

Then the dual program of Eq. (17) is

$$
\begin{aligned}
& \max _{\tilde{C}} \min _{h, \mathbf{h}, \mathfrak{h}} L(\tilde{C}, h, \mathbf{h}, \mathfrak{h})=\max _{\tilde{C}} \min _{h, \mathbf{h}, \mathfrak{h}} 4\left[\mathbf{h}^{\dagger} \mathbf{h}+\operatorname{Tr}\left(\Lambda \mathfrak{h}^{2}\right)\right]+\operatorname{Tr}\left[\tilde{C}\left(H+h \mathbb{1}+\mathbf{J}^{\dagger} \mathbf{h}+\mathbf{h}^{\dagger} \mathbf{J}+\mathbf{J}^{\dagger} \mathfrak{h} \mathbf{J}\right)\right] \\
& =\max _{\substack{\tilde{C}: \operatorname{Tr}(\tilde{C})=0, \mathbf{h}, \mathfrak{h} \\
\operatorname{Tr}(\tilde{C} H) \neq 0}} 4\left[\mathbf{h}^{\dagger} \mathbf{h}+\operatorname{Tr}\left(\Lambda \mathfrak{h}^{2}\right)\right]+\operatorname{Tr}(\tilde{C} H)\left[1+\mathbf{h}^{\dagger} \boldsymbol{j}+\boldsymbol{j}^{\dagger} \mathbf{h}+\operatorname{Tr}\left(\mathfrak{h}^{T} \mathbf{j}\right)\right]
\end{aligned}
$$

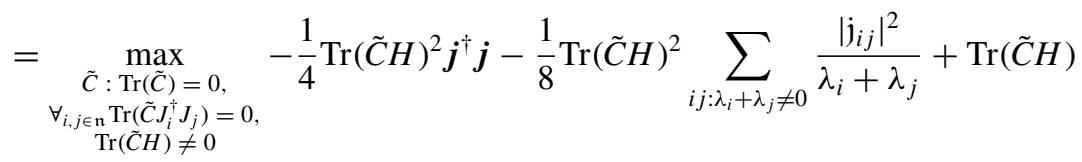

$$
\begin{aligned}
& =\max _{\substack{\tilde{C}: \operatorname{Tr}(\tilde{C})=0, \forall_{i, j \in \mathfrak{n}} \operatorname{Tr}\left(\tilde{C} J_{i}^{\dagger} J_{j}\right)=0,}}\left(\boldsymbol{j}^{\dagger} \boldsymbol{j}+\sum_{i j: \lambda_{i}+\lambda_{j} \neq 0} \frac{\left|\dot{j}_{i j}\right|^{2}}{2\left(\lambda_{i}+\lambda_{j}\right)}\right)^{-1}=\max _{\substack{\tilde{C}: \operatorname{Tr}(\tilde{C})=0, \forall_{i, j \in \mathfrak{n}} \operatorname{Tr}\left(\tilde{C} J_{i}^{\dagger} J_{j}\right)=0}} \mathfrak{F}(C, \tilde{C}),
\end{aligned}
$$

as in Eq. (18). 


\section{APPENDIX D: CONFINING $(h, h)$ IN A COMPACT SET}

The minimax theorem [66] states that for convex compact sets $P \subset \mathbb{R}^{m}$ and $Q \subset \mathbb{R}^{n}$ and $f: P \times Q \rightarrow \mathbb{R}$ such that $f(x, y)$ is a continuous convex (concave) function in $x(y)$ for every fixed $y(x)$, then

$$
\max _{y \in Q} \min _{x \in P} f(x, y)=\min _{x \in P} \max _{y \in Q} f(x, y) .
$$

In Eq. (17), the objective function $4 \operatorname{Tr}\left(C^{\dagger} \alpha C\right)$ is concave (linear) with respect to $C C^{\dagger}$ and convex (quadratic) with respect to $(\mathbf{h}, \mathfrak{h})$. The operator $C C^{\dagger}$ satisfying $\operatorname{Tr}\left(C C^{\dagger}\right)=1$ is contained in a convex compact set, but the domain of $(h, \mathbf{h}, \mathfrak{h})$ is not compact. Here we show that we could always confine $(\mathbf{h}, \mathfrak{h})$ in a convex and compact set such that the solution of Eq. (17) is not altered. As a result, the minimax theorem is applicable and we can exchange the order of the maximization and minimization in Eq. (17).

First we note that $\min _{h, \mathbf{h}, \mathfrak{h} \mid \beta=0}\|\alpha\|=a<\infty$ when $H \in \mathcal{S}$. Note that

$$
\|\alpha\|=\left\|\left(\begin{array}{c}
\mathbf{h}_{1} \mathbb{1}+\sum_{i=1}^{r} \mathfrak{h}_{1 i} L_{i} \\
\mathbf{h}_{2} \mathbb{1}+\sum_{i=1}^{r} \mathfrak{h}_{2 i} L_{i} \\
\vdots \\
\mathbf{h}_{r} \mathbb{1}+\sum_{i=1}^{r} \mathfrak{h}_{r i} L_{i}
\end{array}\right)\right\|^{2} .
$$

It is clear that there exists some $b>0$ such that for all $\|(\mathbf{h}, \mathfrak{h})\|_{2}>b\left(\|\cdot\|_{2}\right.$ is the Euclidean norm), we have $\|\alpha\|>a$. Therefore, it is easy to find some $b^{\prime}>0$ such that

$$
\begin{array}{ll}
\min _{\mathbf{h}, \mathfrak{h}} \max _{C} \quad 4 \operatorname{Tr}\left(C^{\dagger} \alpha C\right), & \\
\text { subject to } \quad \exists h, \beta=0, \quad \operatorname{Tr}\left(C^{\dagger} C\right)=1, \quad\|(\mathbf{h}, \mathfrak{h})\|_{2} \leqslant b^{\prime},
\end{array}
$$

and

$$
\begin{array}{ll}
\max _{C} \min _{\mathbf{h}, \mathfrak{h}} & 4 \operatorname{Tr}\left(C^{\dagger} \alpha C\right), \\
\text { subject to } \quad \exists h, \beta=0, \quad \operatorname{Tr}\left(C^{\dagger} C\right)=1, \quad\|(\mathbf{h}, \mathfrak{h})\|_{2} \leqslant b^{\prime},
\end{array}
$$

has the same optimal value equal to $4 a$, and there exists a saddle point $\left(\mathbf{h}^{*}, \mathfrak{h}^{*}, C^{*}\right)$ such that

$$
\operatorname{Tr}\left(C^{\dagger} \alpha^{*} C\right) \leqslant \operatorname{Tr}\left(C^{* \dagger} \alpha^{*} C^{*}\right) \leqslant \operatorname{Tr}\left(C^{* \dagger} \alpha C^{*}\right)
$$

for all $(\mathbf{h}, \mathfrak{h}, C)$ satisfying $\exists h, \beta=0, \operatorname{Tr}\left(C^{\dagger} C\right)=1$, and $\|(\mathbf{h}, \mathfrak{h})\|_{2} \leqslant b^{\prime}, \quad$ where $\quad \alpha^{*}=\left(\mathbf{h}^{*} \mathbb{1}+\mathfrak{h}^{*} \mathbf{L}\right)^{\dagger}\left(\mathbf{h}^{*} \mathbb{1}+\mathfrak{h}^{*} \mathbf{L}\right)$. Moreover, based on the above discussion, $\left(\mathbf{h}^{*}, \mathfrak{h}^{*}\right)$ is not on the boundary, i.e., $\left\|\left(\mathbf{h}^{*}, \mathfrak{h}^{*}\right)\right\|_{2}<b^{\prime}$. The second inequality in Eq. (D5) is then equivalent to

$$
\operatorname{Re}\left[\operatorname{Tr}\left(C^{* \dagger}(\Delta \mathbf{h} \mathbb{1}+\Delta \mathfrak{h} \mathbf{L})^{\dagger}\left(\mathbf{h}^{*} \mathbb{1}+\mathfrak{h}^{*} \mathbf{L}\right) C^{*}\right)\right]=0,
$$

for all $(\Delta \mathbf{h}, \Delta \mathfrak{h})$ satisfying

$$
\Delta h \mathbb{1}+\Delta \mathbf{h}^{\dagger} \mathbf{L}+\mathbf{L}^{\dagger} \Delta \mathbf{h}+\mathbf{L}^{\dagger} \Delta \mathfrak{h} \mathbf{L}=0
$$

for some $\Delta h$. Therefore, $\left(\mathbf{h}^{*}, \mathfrak{h}^{*}, C^{*}\right)$ is also a saddle point of Eq. (17):

$$
\begin{aligned}
& \max _{C} \min _{\mathbf{h}, \mathfrak{h}} \quad 4 \operatorname{Tr}\left(C^{\dagger} \alpha C\right), \\
& \text { subject to } \quad \exists h, \beta=0, \quad \operatorname{Tr}\left(C^{\dagger} C\right)=1,
\end{aligned}
$$

proving that the optimal value of Eq. (17) must also be equal to $4 a$.

\section{APPENDIX E: THE VALIDITY OF THE NUMERICAL ALGORITHM}

Here we prove the validity of the three-step algorithm introduced in the main text. Let $\left(\mathbf{h}^{*}, \mathfrak{h}^{*}, C^{*}\right)$ be the saddle point of Eq. (17). The first inequality in Eq. (D5) implies

$$
\operatorname{Tr}\left(C^{* \dagger} \alpha^{*} C^{*}\right)=\left\|\alpha^{*}\right\|=\min _{h, \mathbf{h}, \mathfrak{h} \mid \beta=0}\|\alpha\|,
$$

which means that $\Pi^{*} C^{*}=C^{*}$, where $\Pi^{*}$ is the projection onto the subspace spanned by all eigenstates corresponding to the largest eigenvalue of $\alpha^{*}$.

Now assume we have a solution $\left(\mathbf{h}^{\diamond}, \mathfrak{h}^{\diamond}\right)$ of Eq. (2) such that $\alpha^{\diamond}=\left(\mathbf{h}^{\diamond} \mathbb{1}+\mathfrak{h}^{\diamond} \mathbf{L}\right)^{\dagger}\left(\mathbf{h}^{\diamond} \mathbb{1}+\mathfrak{h}^{\diamond} \mathbf{L}\right)$ satisfies

$$
\left\|\alpha^{\diamond}\right\|=\min _{h, \mathbf{h}, \mathfrak{h} \mid \beta=0}\|\alpha\| .
$$

We prove that $\left(\mathbf{h}^{\diamond}, \mathfrak{h}^{\diamond}, C^{*}\right)$ is also a saddle point. Choose $p \in$ $(0,1)$ and let

$$
(\mathbf{h}, \mathfrak{h})=\left[p \mathbf{h}^{\diamond}+(1-p) \mathbf{h}^{*}, p \mathfrak{h}^{\diamond}+(1-p) \mathfrak{h}^{*}\right] .
$$

Then,

$$
\begin{aligned}
\operatorname{Tr}\left(C^{* \dagger} \alpha C^{*}\right) & =p^{2} \operatorname{Tr}\left(C^{* \dagger} \alpha^{\diamond} C^{*}\right)+(1-p)^{2} \operatorname{Tr}\left(C^{* \dagger} \alpha^{*} C^{*}\right)+2 p(1-p) \operatorname{Re}\left\{\operatorname{Tr}\left[C^{* \dagger}\left(\mathbf{h}^{\diamond} \mathbb{1}+\mathfrak{h}^{\diamond} \mathbf{L}\right)^{\dagger}\left(\mathbf{h}^{*} \mathbb{1}+\mathfrak{h}^{*} \mathbf{L}\right) C^{*}\right]\right\} \\
& \leqslant p^{2} \operatorname{Tr}\left(C^{* \dagger} \alpha^{\diamond} C^{*}\right)+(1-p)^{2} \operatorname{Tr}\left(C^{* \dagger} \alpha^{*} C^{*}\right)+2 p(1-p) \sqrt{\operatorname{Tr}\left(C^{* \dagger} \alpha^{\diamond} C^{*}\right) \operatorname{Tr}\left(C^{* \dagger} \alpha^{*} C^{*}\right)} \leqslant\left\|\alpha^{*}\right\| .
\end{aligned}
$$

On the other hand, we know $\operatorname{Tr}\left(C^{* \dagger} \alpha C^{*}\right) \geqslant\left\|\alpha^{*}\right\|$. Therefore, the equality in Eq. (E4) must hold, which means

$$
\operatorname{Tr}\left(C^{* \dagger} \alpha^{\diamond} C^{*}\right)=\left\|\alpha^{\diamond}\right\|, \quad\left(\mathbf{h}^{*} \mathbb{1}+\mathfrak{h}^{*} \mathbf{L}\right) C^{*}=\left(\mathbf{h}^{\diamond} \mathbb{1}+\mathfrak{h}^{\diamond} \mathbf{L}\right) C^{*} .
$$

As a result, we have $\operatorname{Tr}\left(C^{\dagger} \alpha^{\diamond} C\right) \leqslant \operatorname{Tr}\left(C^{* \dagger} \alpha^{\diamond} C^{*}\right)$ for arbitrary $C$ satisfying $\operatorname{Tr}\left(C^{\dagger} C\right)=1$. Moreover,

$$
\operatorname{Re}\left\{\operatorname{Tr}\left[C^{* \dagger}(\Delta \mathbf{h} \mathbb{1}+\Delta \mathfrak{h} \mathbf{L})^{\dagger}\left(\mathbf{h}^{\diamond} \mathbb{1}+\mathfrak{h}^{\diamond} \mathbf{L}\right) C^{*}\right]\right\}=\operatorname{Re}\left\{\operatorname{Tr}\left[C^{* \dagger}(\Delta \mathbf{h} \mathbb{1}+\Delta \mathfrak{h} \mathbf{L})^{\dagger}\left(\mathbf{h}^{*} \mathbb{1}+\mathfrak{h}^{*} \mathbf{L}\right) C^{*}\right]\right\}=0,
$$

and $\operatorname{Tr}\left(C^{* \dagger} \alpha^{\diamond} C^{*}\right) \leqslant \operatorname{Tr}\left(C^{* \dagger} \alpha C^{*}\right)$, proving $\left(\mathbf{h}^{\diamond}, \mathfrak{h}^{\diamond}, C^{*}\right)$ is also a saddle point. Hence, step (b) in our algorithm will at least have one solution $C^{*}$, and the solution of step (b) $\left(\mathbf{h}^{\diamond}, \mathfrak{h}^{\diamond}, C^{\diamond}\right)$ is also a saddle point satisfying

$$
\operatorname{Tr}\left(C^{\dagger} \alpha^{\diamond} C\right) \leqslant \operatorname{Tr}\left(C^{\diamond \dagger} \alpha^{\diamond} C^{\diamond}\right) \leqslant \operatorname{Tr}\left(C^{\diamond \dagger} \alpha C^{\diamond}\right),
$$


for all $(h, \mathbf{h}, \mathfrak{h}, C)$ satisfying $\beta=0$ and $\operatorname{Tr}\left(C^{\dagger} C\right)=1$. Strong duality [64] implies the optimal value of

$\max _{\tilde{C}} \mathfrak{F}\left(C^{\diamond}, \tilde{C}\right), \quad$ subject to $\operatorname{Tr}\left(C^{\dagger} C\right)=1, \quad \operatorname{Tr}(\tilde{C})=0$,

$$
\text { and } \operatorname{Tr}\left(J_{i}^{\dagger} J_{j} \tilde{C}\right)=0 \quad \forall i, j \in \mathfrak{n}, \quad(\mathrm{E} 8)
$$

is equal to that of $\min _{h, \mathbf{h}, \mathfrak{h} \mid \beta=0} 4 \operatorname{Tr}\left(C^{\diamond \dagger} \alpha C^{\diamond}\right)=$ $\min _{h, \mathbf{h}, \mathfrak{h} \mid \beta=0}\|\alpha\|$, proving the optimality of $\left(C^{\diamond}, \tilde{C}^{\diamond}\right)$.

\section{APPENDIX F: HIGHLY BIASED NOISE}

We derived the optimal $\mathfrak{F}$ and the corresponding optimal AQEC code under the highly biased noise model, taking the limit $\eta \rightarrow 0$. Using the highly biased model [Eq. (23)], we need to replace $\mathbf{L}$ by $\left(\Pi_{\mathfrak{s}}+\Pi_{\overline{\mathfrak{s}}} \sqrt{\eta}\right) \mathbf{L}$ in Eq. (2), where $\Pi_{\overline{\mathfrak{s}} \text { (or } \mathfrak{s})}$ is an $r$-by- $r$ diagonal matrix whose $i$ th diagonal element is one when $i \in \overline{\mathfrak{s}}($ or $\mathfrak{s})$, and zero when $i \in \mathfrak{s}($ or $\overline{\mathfrak{s}})$. After the following parameter transformation:

$$
\begin{aligned}
& \mathbf{h} \leftarrow\left(\Pi_{\mathfrak{s}}+\Pi_{\overline{\mathfrak{s}}} / \sqrt{\eta}\right) \mathbf{h}, \\
& \mathfrak{h} \leftarrow\left(\Pi_{\mathfrak{s}}+\Pi_{\overline{\mathfrak{s}}} / \sqrt{\eta}\right) \mathfrak{h}\left(\Pi_{\mathfrak{s}}+\Pi_{\overline{\mathfrak{s}}} / \sqrt{\eta}\right),
\end{aligned}
$$

in Eq. (2), we have that the optimal QFI is equal to $\mathfrak{F}^{\diamond}=$ $\min _{h, \mathbf{h}, \mathfrak{h} \mid \beta=0}\|\alpha\|$, where

$$
\begin{gathered}
\alpha=(\mathbf{h} \mathbb{1}+\mathfrak{h} \mathbf{L})^{\dagger}\left(\Pi_{\mathfrak{s}}+\Pi_{\overline{\mathfrak{s}}} / \eta\right)(\mathbf{h} \mathbb{1}+\mathfrak{h} \mathbf{L}), \\
\beta=H+h \mathbb{1}+\mathbf{h}^{\dagger} \mathbf{L}+\mathbf{L}^{\dagger} \mathbf{h}+\mathbf{L}^{\dagger} \mathfrak{h} \mathbf{L} .
\end{gathered}
$$

Letting $\bar{\alpha}=(\mathbf{h} \mathbb{1}+\mathfrak{h} \mathbf{L})^{\dagger} \Pi_{\overline{\mathfrak{s}}}(\mathbf{h} \mathbb{1}+\mathfrak{h} \mathbf{L})$, we have

$$
\mathfrak{F}^{\diamond}=\frac{1}{\eta} \min _{h, \mathbf{h}, \mathfrak{h} \mid \beta=0}\|\bar{\alpha}\|+O(1),
$$

where $\quad \min _{h, \mathbf{h}, \mathfrak{h} \mid \beta=0}\|\bar{\alpha}\|>0 \quad$ as $\quad$ long $\quad$ as $\quad H \notin$ $\operatorname{span}\left\{\mathbb{1}, L_{i}, L_{i}^{\dagger}, L_{i}^{\dagger} L_{j}, i, j \in \mathfrak{s}\right\}$.

Now consider the dual program of the modified version of Eq. (17) with $\alpha$ replaced by $\bar{\alpha}$. We first simplify the calculation by performing a gauge transformation such that the new set of Lindblad operators $\mathbf{J}$ satisfies $\operatorname{Tr}\left(C^{\dagger} J_{i} C\right)=0$, $\mathscr{J}_{\mathfrak{s i n}}$ is diagonal with the $i$ th diagonal element equal to $\lambda_{i}$ when $i \in \mathfrak{s}$ and zero when $i \in \overline{\mathfrak{s}}$, and its Schur complement $\mathscr{J}_{\overline{\mathfrak{s}} \overline{\mathfrak{s}}}-\mathscr{J}_{\overline{\mathfrak{s} s}} \mathscr{J}_{\mathfrak{s \mathfrak { s }}}^{-1} \mathscr{J}_{\mathfrak{s} \bar{s}}$ is diagonal with the $i$ th diagonal element equal to $\lambda_{i}$ when $i \in \overline{\mathfrak{s}}$ and zero when $i \in \mathfrak{s}$. Here, the superscript ${ }^{-1}$ means the Moore-Penrose pseudoinverse and we use the notations $(\cdot) \square=\Pi_{\square}(\cdot) \Pi_{\square}$ for $\square, \boldsymbol{\square}=\overline{\mathfrak{s}}, \mathfrak{s}$ and $\mathscr{J}_{i j}=\operatorname{Tr}\left(C^{\dagger} J_{i}^{\dagger} J_{j} C\right)$. Note that the gauge transformation here is divided into two steps: (1) $L_{i} \leftarrow L_{i}-\operatorname{Tr}\left(C^{\dagger} L_{i} C\right) \mathbb{1}$ and (2) $\mathbf{L} \leftarrow\left(u \Pi_{\mathfrak{s}}+v \Pi_{\overline{\mathfrak{s}}}\right) \mathbf{L}$, where $u=\Pi_{\mathfrak{s}} u \Pi_{\mathfrak{s}}$ and $v=\Pi_{\overline{\mathfrak{s}}} v \Pi_{\overline{\mathfrak{s}}}$ are some unitary operators within the subspaces defined by $\Pi_{\mathfrak{s}}$ and $\Pi_{\mathfrak{s}}$. In this way, the solution is invariant. Again, we introduce a Hermitian matrix $\tilde{C}$ as a Lagrange multiplier, and the Lagrange function is

$$
L(\tilde{C}, h, \mathbf{h}, \mathfrak{h})=4 \operatorname{Tr}\left[C^{\dagger}(\mathbf{h} \mathbb{1}+\mathfrak{h} \mathbf{J})^{\dagger} \Pi_{\overline{\mathfrak{s}}}(\mathbf{h} \mathbb{1}+\mathfrak{h} \mathbf{J}) C\right]+\operatorname{Tr}\left[\tilde{C}\left(H+h \mathbb{1}+\mathbf{J}^{\dagger} \mathbf{h}+\mathbf{h}^{\dagger} \mathbf{J}+\mathbf{J}^{\dagger} \mathfrak{h} \mathbf{J}\right)\right]
$$

Then we have

$$
\begin{aligned}
\min _{h, \mathbf{h}, \mathfrak{h} \mid \beta=0}\|\bar{\alpha}\| & =\max _{\tilde{C}} \min _{h, \mathbf{h}, \mathfrak{h}} L(\tilde{C}, h, \mathbf{h}, \mathfrak{h}) \\
& =\max _{\tilde{C}: \operatorname{Tr}(\tilde{C})=0} \min _{\mathbf{h}, \mathfrak{h}} 4\left[\mathbf{h}^{\dagger} \Pi_{\overline{\mathfrak{s}}} \mathbf{h}+\operatorname{Tr}\left(\mathfrak{h}^{\dagger} \Pi_{\bar{s}} \mathfrak{h} \mathscr{J}^{T}\right)\right]+\operatorname{Tr}(\tilde{C} H)\left[1+\mathbf{h}^{\dagger} \boldsymbol{j}+\boldsymbol{j}^{\dagger} \mathbf{h}+\operatorname{Tr}\left(\mathfrak{h} \mathfrak{j}^{T}\right)\right] \\
& =\max _{\substack{\tilde{C}: \operatorname{Tr}(\tilde{C})=0, \operatorname{Tr}(\tilde{C} H) \neq 0, \Pi_{\mathfrak{s}} j=0, \mathfrak{j}_{\mathfrak{s} \mathfrak{s}}=0}}\left[-\frac{1}{4} \operatorname{Tr}(\tilde{C} H)^{2} \boldsymbol{j}^{\dagger} \Pi_{\overline{\mathfrak{s}}} \boldsymbol{j}+\operatorname{Tr}(\tilde{C} H)+(*)\right],
\end{aligned}
$$

and

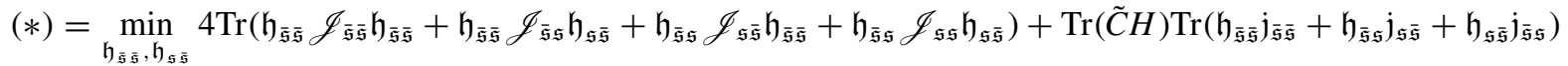

$$
\begin{aligned}
& =\min _{\mathfrak{h}_{\overline{\mathfrak{s}} \overline{\mathfrak{s}}}} 4 \operatorname{Tr}\left[\mathfrak{h}_{\overline{\mathfrak{s}} \overline{\mathfrak{s}}}\left(\mathscr{J}_{\overline{\mathfrak{s}} \overline{\mathfrak{s}}}-\mathscr{J}_{\overline{\mathfrak{s}} \mathfrak{s}} \mathscr{J}_{\mathfrak{s} \mathfrak{s}}^{-1} \mathscr{J}_{\mathfrak{s} \overline{\mathfrak{s}}}\right) \mathfrak{h}_{\overline{\mathfrak{s}} \overline{\mathfrak{s}}}\right] \\
& +\operatorname{Tr}(\tilde{C} H) \operatorname{Tr}\left[\mathfrak{h}_{\overline{\mathfrak{s}} \mathfrak{s}}\left(\mathfrak{j}_{\overline{\mathfrak{s}} \overline{\mathfrak{s}}}-\mathscr{J}_{\overline{\mathfrak{s}} \mathfrak{s}} \mathscr{J}_{\mathfrak{s} \mathfrak{s}}^{-1} \mathfrak{j}_{\mathfrak{s} \mathfrak{s}}-\mathfrak{j}_{\overline{\mathfrak{s}} \mathfrak{s}} \mathscr{J}_{\mathfrak{s} \mathfrak{s}}^{-1} \mathscr{J}_{\mathfrak{s} \overline{\mathfrak{s}}}\right)\right]-\frac{\operatorname{Tr}(\tilde{C} H)^{2}}{4} \operatorname{Tr}\left(\mathfrak{j}_{\overline{\mathfrak{s}} \mathfrak{s}} \mathscr{J}_{\mathfrak{s} \mathfrak{s}}^{-1} \mathfrak{j}_{\mathfrak{s} \overline{\mathfrak{s}}}\right)
\end{aligned}
$$

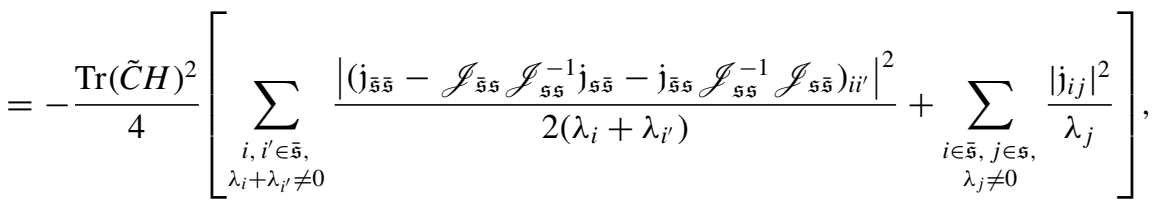

under the constraints that $\Pi_{\mathfrak{n}_{\mathfrak{s}}} \mathfrak{j}_{\mathfrak{s s}}=0, \Pi_{\mathfrak{n}_{\mathfrak{s}}} \mathscr{J}_{\mathfrak{s} \overline{\mathfrak{s}}}=0$,

$$
\Pi_{\mathfrak{n}_{\overline{\mathfrak{s}}}}\left(\mathfrak{j}_{\overline{\mathfrak{s}} \overline{\mathfrak{s}}}-\mathscr{J}_{\overline{\mathfrak{s}} \mathfrak{s}} \mathscr{J}_{\mathfrak{s s}}^{-1} \mathfrak{j}_{\mathfrak{s} \overline{\mathfrak{s}}}-\mathfrak{j}_{\overline{\mathfrak{s}} \mathfrak{s}} \mathscr{J}_{\mathfrak{s s}}^{-1} \mathscr{J}_{\mathfrak{s} \overline{\mathfrak{s}}}\right) \Pi_{\mathfrak{n}_{\overline{\mathfrak{s}}}}=0,
$$

where $\Pi_{\mathfrak{n}_{\mathfrak{s}}, \mathfrak{n}_{\overline{\mathfrak{s}}}}$ are projection operators defined by $\mathfrak{n}_{\mathfrak{s}}=\left\{i \in \mathfrak{s} \mid \lambda_{i}=0\right\}, \mathfrak{n}_{\overline{\mathfrak{s}}}=\left\{i \in \overline{\mathfrak{s}} \mid \lambda_{i}=0\right\}$. Otherwise, $(*)=-\infty$. Note that the second constraint $\Pi_{\mathfrak{n}_{\mathfrak{s}}} \mathscr{J}_{\mathfrak{s} \overline{\mathfrak{s}}}=0$ is automatically satisfied by definition. 
To conclude, the dual program after replacing $\alpha$ by $\bar{\alpha}$ is equal to

$$
\begin{aligned}
\max _{C, \tilde{C}} & \overline{\mathfrak{F}}(C, \tilde{C}), \quad \text { subject to } \operatorname{Tr}\left(C^{\dagger} C\right)=1, \quad \operatorname{Tr}(\tilde{C})=0, \quad \operatorname{Tr}(\tilde{C} H) \neq 0, \\
& \Pi_{\mathfrak{s}} \boldsymbol{j}=0, \quad \mathfrak{j}_{\mathfrak{s s}}=0, \quad \Pi_{\mathfrak{n}_{\mathfrak{s}}} \mathfrak{j}_{\mathfrak{s} \bar{s}}=0, \quad \Pi_{\mathfrak{n}_{\overline{\mathfrak{s}}}}\left(\mathfrak{j}_{\overline{\mathfrak{s}} \overline{\mathfrak{s}}}-\mathscr{J}_{\overline{\mathfrak{s}} \mathfrak{s}} \mathscr{J}_{\mathfrak{s s}}^{-1} \mathfrak{j}_{\mathfrak{s} \bar{s}}-\mathfrak{j}_{\overline{\mathfrak{s}} \mathfrak{s}} \mathscr{J}_{\mathfrak{s i s}}^{-1} \mathscr{J}_{\mathfrak{s} \bar{s}}\right) \Pi_{\mathfrak{n}_{\overline{\mathfrak{s}}}}=0,
\end{aligned}
$$

where

$$
\overline{\mathfrak{F}}(C, \tilde{C})=\left[\boldsymbol{j}^{\dagger} \Pi_{\overline{\mathfrak{s}}} \boldsymbol{j}+\sum_{\substack{i, i^{\prime} \in \overline{\mathfrak{s}}, \lambda_{i}+\lambda_{i^{\prime}} \neq 0}} \frac{\left|\left(\mathfrak{j}_{\overline{\mathfrak{s}} \overline{\mathfrak{s}}}-\mathscr{J}_{\overline{\mathfrak{s}} \mathfrak{s}} \mathscr{J}_{\mathfrak{s} \mathfrak{s}}^{-1} \dot{j}_{\mathfrak{s} \overline{\mathfrak{s}}}-\mathfrak{j}_{\overline{\mathfrak{s}} \mathfrak{s}} \mathscr{J}_{\mathfrak{s} \mathfrak{s}}^{-1} \mathscr{J}_{\mathfrak{s} \mathfrak{s}}\right)_{i i^{\prime}}\right|^{2}}{2\left(\lambda_{i}+\lambda_{i^{\prime}}\right)}+\sum_{\substack{i \in \overline{\mathfrak{s}}, j \in \mathfrak{s}, \lambda_{j} \neq 0}} \frac{\left|\dot{j}_{i j}\right|^{2}}{\lambda_{j}}\right]^{-1} .
$$

\section{APPENDIX G: THE OPTIMAL AQEC CODE FOR CORRELATED-DEPHASING NOISE}

In this Appendix, we provide a nonperturbation QEC code achieving the optimal $\mathfrak{F}$ in a correlated noise-dephasing noise model [32,33]. We have $N \geqslant 3$ qubits evolving under

$$
\begin{aligned}
\frac{d \rho}{d t} & =-i \omega[\boldsymbol{w} \cdot \boldsymbol{Z}, \rho]+\frac{1}{2} \sum_{j k}\left(\Gamma_{j k} Z_{j} \rho Z_{k}-\frac{1}{2}\left\{Z_{k} Z_{j}, \rho\right\}\right) \\
& =-i \omega[\boldsymbol{w} \cdot \boldsymbol{Z}, \rho]+\sum_{j} \frac{\mu_{j}}{2}\left[\left(\boldsymbol{v}_{j} \cdot \boldsymbol{Z}\right) \rho\left(\boldsymbol{v}_{j} \cdot \boldsymbol{Z}\right)-\frac{1}{2}\left\{\left(\boldsymbol{v}_{j} \cdot \boldsymbol{Z}\right)^{2}, \rho\right\}\right]
\end{aligned}
$$

where $Z_{i}$ is the Pauli-Z operator on the $i$ th qubit, $\boldsymbol{w}$ and $\boldsymbol{v}_{i}$ are all unit vectors, and $\Gamma=\sum_{i} \mu_{i} \boldsymbol{v}_{i} \boldsymbol{v}_{i}^{T}$, with $\mu_{i}>0$ and $\left\{v_{i}\right\}$ an orthonormal set of vectors. The violation of the HNLS condition $H \in \mathcal{S}$ is equivalent to $\boldsymbol{w} \in \operatorname{span}\left\{\boldsymbol{v}_{i}, \forall i\right\}$.

We first calculate the optimal normalized QFI,

$$
4 \min _{h, \mathbf{h}, \mathfrak{h} \mid \beta=0}\|\alpha\|=4 \mathbf{h}^{\dagger} \mathbf{h}=2 \sum_{i} \frac{\left(\boldsymbol{v}_{i} \cdot \boldsymbol{w}\right)^{2}}{\mu_{i}}=2 \boldsymbol{w}^{T} \Gamma^{-1} \boldsymbol{w},
$$

where the superscript ${ }^{-1}$ means the Moore-Penrose pseudoinverse, and

$$
\begin{aligned}
\beta & =H+h \mathbb{1}+\mathbf{h}^{\dagger} \mathbf{L}+\mathbf{L}^{\dagger} \mathbf{h}+\mathbf{L}^{\dagger} \mathfrak{h} \mathbf{L} \\
& =\boldsymbol{w} \cdot \boldsymbol{Z}+\left(\begin{array}{llll}
\mathbb{1} & \sqrt{\frac{\mu_{1}}{2}} \boldsymbol{v}_{1} \cdot \boldsymbol{Z} & \cdots & \sqrt{\frac{\mu_{N}}{2}} \boldsymbol{v}_{N} \cdot \boldsymbol{Z}
\end{array}\right)\left(\begin{array}{cc}
h & \mathbf{h}^{\dagger} \\
\mathbf{h} & \mathfrak{h}
\end{array}\right)\left(\begin{array}{c}
\mathbb{1} \\
\sqrt{\frac{\mu_{1}}{2}} \boldsymbol{v}_{1} \cdot \boldsymbol{Z} \\
\vdots \\
\sqrt{\frac{\mu_{N}}{2}} \boldsymbol{v}_{N} \cdot \boldsymbol{Z}
\end{array}\right) \\
& =\boldsymbol{w} \cdot \boldsymbol{Z}+\sum_{i}\left(\mathbf{h}_{i}+\mathbf{h}_{i}^{\dagger}\right) \sqrt{\frac{\mu_{i}}{2}} \boldsymbol{v}_{i} \cdot \boldsymbol{Z}=0 \Rightarrow \mathbf{h}_{i}=\frac{\boldsymbol{v}_{i} \cdot \boldsymbol{w}}{\sqrt{2 \mu_{i}}}, \quad \mathfrak{h}=0 .
\end{aligned}
$$

Now we introduce a QEC code,

$$
\left|0_{\mathrm{L}}\right\rangle=\bigotimes_{j=1}^{N}\left(\cos \theta_{j}\left|0_{j}\right\rangle+i \sin \theta_{j}\left|1_{j}\right\rangle\right), \quad\left|1_{\mathrm{L}}\right\rangle=X^{\otimes N}\left|0_{\mathrm{L}}\right\rangle,
$$

where $\boldsymbol{\theta}=\frac{1}{2} \arccos \chi \boldsymbol{u}$, defined elementwise, satisfying

$$
P(\boldsymbol{v} \cdot \boldsymbol{Z}) P=\boldsymbol{v} \cdot \cos (2 \boldsymbol{\theta}) Z_{\mathrm{L}}=\chi(\boldsymbol{v} \cdot \boldsymbol{u}) Z_{\mathrm{L}},
$$

and $P(\boldsymbol{v} \cdot \boldsymbol{Z})\left(\boldsymbol{v}^{\prime} \cdot \boldsymbol{Z}\right) P \propto P$ for any $\boldsymbol{v}$ and $\boldsymbol{v}^{\prime} . \chi$ is a tunable parameter $\in\left(0,\|\boldsymbol{u}\|_{\infty}^{-1}\right]$, where $\|\cdot\|_{\infty}$ is the infinity norm.

The QEC code is designed to correct every mode $\boldsymbol{v}$ perpendicular to $\boldsymbol{u}$. Using the recovery channel introduced in Appendix $\mathrm{F}$ of [33], we would have an effective channel

$$
\frac{d \rho}{d t}=-i\left[\omega \chi\left(\boldsymbol{u}^{T} \boldsymbol{w}\right) Z_{\mathrm{L}}, \rho\right]+\frac{\left(\boldsymbol{u}^{T} \Gamma \boldsymbol{u}\right) \chi^{2}}{2}\left(Z_{\mathrm{L}} \rho Z_{\mathrm{L}}-\rho\right) .
$$

Using spin-squeezed states as input states, we could achieve the optimal $\mathfrak{F}$ because

$$
\mathfrak{F}=\frac{4 \chi^{2}\left(\boldsymbol{u}^{T} \boldsymbol{w}\right)^{2}}{2 \chi^{2}\left(\boldsymbol{u}^{T} \Gamma \boldsymbol{u}\right)} \leqslant 2 \boldsymbol{w} \Gamma^{-1} \boldsymbol{w}
$$


where the second equality holds when

$$
\left(\boldsymbol{u}^{T} \Gamma \boldsymbol{u}\right)\left(\boldsymbol{w}^{T} \Gamma^{-1} \boldsymbol{w}\right)=\left(\boldsymbol{u}^{T} \boldsymbol{w}\right)^{2} \Leftrightarrow \boldsymbol{u} \propto \Gamma^{-1} \boldsymbol{w} .
$$

Note that $\mathfrak{F}=2 \boldsymbol{w} \Gamma^{-1} \boldsymbol{w}$ could be very large if there exists an $i$ such that $\mu_{i} \ll\left(\boldsymbol{v}_{i} \cdot \boldsymbol{w}\right)^{2}$.

[1] V. Giovannetti, S. Lloyd, and L. Maccone, Quantum Metrology, Phys. Rev. Lett. 96, 010401 (2006).

[2] V. Giovannetti, S. Lloyd, and L. Maccone, Advances in quantum metrology, Nat. Photon. 5, 222 (2011).

[3] C. L. Degen, F. Reinhard, and P. Cappellaro, Quantum sensing, Rev. Mod. Phys. 89, 035002 (2017).

[4] D. Braun, G. Adesso, F. Benatti, R. Floreanini, U. Marzolino, M. W. Mitchell, and S. Pirandola, Quantum-enhanced measurements without entanglement, Rev. Mod. Phys. 90, 035006 (2018).

[5] L. Pezzè, A. Smerzi, M. K. Oberthaler, R. Schmied, and P. Treutlein, Quantum metrology with nonclassical states of atomic ensembles, Rev. Mod. Phys. 90, 035005 (2018).

[6] S. Pirandola, B. R. Bardhan, T. Gehring, C. Weedbrook, and S. Lloyd, Advances in photonic quantum sensing, Nat. Photon. 12, 724 (2018).

[7] C. M. Caves, Quantum-mechanical noise in an interferometer, Phys. Rev. D 23, 1693 (1981).

[8] D. J. Wineland, J. J. Bollinger, W. M. Itano, F. Moore, and D. Heinzen, Spin squeezing and reduced quantum noise in spectroscopy, Phys. Rev. A 46, R6797 (1992).

[9] M. Kitagawa and M. Ueda, Squeezed spin states, Phys. Rev. A 47, 5138 (1993).

[10] S. F. Huelga, C. Macchiavello, T. Pellizzari, A. K. Ekert, M. B. Plenio, and J. I. Cirac, Improvement of Frequency Standards with Quantum Entanglement, Phys. Rev. Lett. 79, 3865 (1997).

[11] D. Ulam-Orgikh and M. Kitagawa, Spin squeezing and decoherence limit in Ramsey spectroscopy, Phys. Rev. A 64, 052106 (2001).

[12] R. Demkowicz-Dobrzański, K. Banaszek, and R. Schnabel, Fundamental quantum interferometry bound for the squeezedlight-enhanced gravitational wave detector GEO 600, Phys. Rev. A 88, 041802 (2013).

[13] R. Chaves, J. Brask, M. Markiewicz, J. Kołodyński, and A. Acín, Noisy Metrology Beyond the Standard Quantum Limit, Phys. Rev. Lett. 111, 120401 (2013).

[14] M. B. Plenio and S. F. Huelga, Sensing in the presence of an observed environment, Phys. Rev. A 93, 032123 (2016).

[15] F. Albarelli, M. A. Rossi, M. G. Paris, and M. G. Genoni, Ultimate limits for quantum magnetometry via time-continuous measurements, New J. Phys. 19, 123011 (2017).

[16] F. Albarelli, M. A. Rossi, D. Tamascelli, and M. G. Genoni, Restoring Heisenberg scaling in noisy quantum metrology by monitoring the environment, Quantum 2, 110 (2018).

[17] Y. Matsuzaki, S. C. Benjamin, and J. Fitzsimons, Magnetic field sensing beyond the standard quantum limit under the effect of decoherence, Phys. Rev. A 84, 012103 (2011).

[18] A. W. Chin, S. F. Huelga, and M. B. Plenio, Quantum Metrology in Non-Markovian Environments, Phys. Rev. Lett. 109, 233601 (2012).

[19] A. Smirne, J. Kołodyński, S. F. Huelga, and R. DemkowiczDobrzański, Ultimate Precision Limits for Noisy Frequency Estimation, Phys. Rev. Lett. 116, 120801 (2016).
[20] H. Yuan, Sequential Feedback Scheme Outperforms the Parallel Scheme for Hamiltonian Parameter Estimation, Phys. Rev. Lett. 117, 160801 (2016).

[21] J. Liu and H. Yuan, Quantum parameter estimation with optimal control, Phys. Rev. A 96, 012117 (2017).

[22] H. Xu, J. Li, L. Liu, Y. Wang, H. Yuan, and X. Wang, Generalizable control for quantum parameter estimation through reinforcement learning, npj Quantum Inf. 5, 82 (2019).

[23] E. M. Kessler, I. Lovchinsky, A. O. Sushkov, and M. D. Lukin, Quantum Error Correction for Metrology, Phys. Rev. Lett. 112, 150802 (2014).

[24] G. Arrad, Y. Vinkler, D. Aharonov, and A. Retzker, Increasing Sensing Resolution with Error Correction, Phys. Rev. Lett. 112, 150801 (2014).

[25] W. Dür, M. Skotiniotis, F. Froewis, and B. Kraus, Improved Quantum Metrology Using Quantum Error Correction, Phys. Rev. Lett. 112, 080801 (2014).

[26] R. Ozeri, Heisenberg limited metrology using quantum errorcorrection codes, arXiv:1310.3432.

[27] F. Reiter, A. S. Sørensen, P. Zoller, and C. Muschik, Dissipative quantum error correction and application to quantum sensing with trapped ions, Nat. Commun. 8, 1822 (2017).

[28] X.-M. Lu, S. Yu, and C. Oh, Robust quantum metrological schemes based on protection of quantum fisher information, Nat. Commun. 6, 7282 (2015).

[29] P. Sekatski, M. Skotiniotis, J. Kołodyński, and W. Dür, Quantum metrology with full and fast quantum control, Quantum 1, 27 (2017).

[30] R. Demkowicz-Dobrzański, J. Czajkowski, and P. Sekatski, Adaptive Quantum Metrology Under General Markovian Noise, Phys. Rev. X 7, 041009 (2017).

[31] S. Zhou, M. Zhang, J. Preskill, and L. Jiang, Achieving the Heisenberg limit in quantum metrology using quantum error correction, Nat. Commun. 9, 78 (2018).

[32] D. Layden and P. Cappellaro, Spatial noise filtering through error correction for quantum sensing, npj Quantum Inf. 4, 30 (2018).

[33] D. Layden, S. Zhou, P. Cappellaro, and L. Jiang, AncillaFree Quantum Error Correction Codes for Quantum Metrology, Phys. Rev. Lett. 122, 040502 (2019).

[34] W. Gorecki, S. Zhou, L. Jiang, and R. Demkowicz-Dobrzanski, Optimal probes and error-correction schemes in multiparameter quantum metrology, arXiv:1901.00896.

[35] T. Kapourniotis and A. Datta, Fault-tolerant quantum metrology, Phys. Rev. A 100, 022335 (2019).

[36] Q. Zhuang, J. Preskill, and L. Jiang, Distributed quantum sensing enhanced by continuous-variable error correction, arXiv:1910.14156.

[37] D. Leibfried, M. D. Barrett, T. Schaetz, J. Britton, J. Chiaverini, W. M. Itano, J. D. Jost, C. Langer, and D. J. Wineland, Toward Heisenberg-limited spectroscopy with multiparticle entangled states, Science 304, 1476 (2004). 
[38] A. Fujiwara and H. Imai, A fibre bundle over manifolds of quantum channels and its application to quantum statistics, J. Phys. A: Math. Theor. 41, 255304 (2008).

[39] B. Escher, R. de Matos Filho, and L. Davidovich, General framework for estimating the ultimate precision limit in noisy quantum-enhanced metrology, Nat. Phys. 7, 406 (2011).

[40] Z. Ji, G. Wang, R. Duan, Y. Feng, and M. Ying, Parameter estimation of quantum channels, IEEE Trans. Inf. Theory $\mathbf{5 4}$, 5172 (2008).

[41] R. Demkowicz-Dobrzański, J. Kołodyński, and M. Guţă, The elusive Heisenberg limit in quantum-enhanced metrology, Nat. Commun. 3, 1063 (2012).

[42] R. Demkowicz-Dobrzański and L. Maccone, Using Entanglement Against Noise in Quantum Metrology, Phys. Rev. Lett. 113, 250801 (2014).

[43] J. Kołodyński and R. Demkowicz-Dobrzański, Efficient tools for quantum metrology with uncorrelated noise, New J. Phys. 15, 073043 (2013).

[44] J. Czajkowski, K. Pawłowski, and R. Demkowicz-Dobrzański, Many-body effects in quantum metrology, New J. Phys. 21, 053031 (2019).

[45] S. Pirandola and C. Lupo, Ultimate Precision of Adaptive Noise Estimation, Phys. Rev. Lett. 118, 100502 (2017).

[46] R. Laurenza, C. Lupo, G. Spedalieri, S. L. Braunstein, and S. Pirandola, Channel simulation in quantum metrology, Quantum Meas. Quantum Metrol. 5, 1 (2018).

[47] M. A. Nielsen and I. Chuang, Quantum Computation and Quantum Information (Cambridge University Press, Cambridge, England, 2000).

[48] V. Gorini, A. Kossakowski, and E. C. G. Sudarshan, Completely positive dynamical semigroups of n-level systems, J. Math. Phys. 17, 821 (1976).

[49] G. Lindblad, On the generators of quantum dynamical semigroups, Commun. Math. Phys. 48, 119 (1976).

[50] H.-P. Breuer, F. Petruccione et al., The Theory of Open Quantum Systems (Oxford University Press, Oxford, England, 2002).

[51] C. Helstrom, The minimum variance of estimates in quantum signal detection, IEEE Trans. Inf. Theory 14, 234 (1968).

[52] C. W. Helstrom, Quantum Detection and Estimation Theory (Academic Press, New York, 1976).

[53] S. L. Braunstein and C. M. Caves, Statistical Distance and the Geometry of Quantum States, Phys. Rev. Lett. 72, 3439 (1994).
[54] M. G. Paris, Quantum estimation for quantum technology, Int. J. Quantum Inf. 7, 125 (2009).

[55] G. Casella and R. L. Berger, Statistical Inference (Duxbury, Pacific Grove, CA, 2002), Vol. 2.

[56] E. L. Lehmann and G. Casella, Theory of Point Estimation (Springer Science \& Business Media, New York, 2006).

[57] H. Barnum and E. Knill, Reversing quantum dynamics with near-optimal quantum and classical fidelity, J. Math. Phys. 43, 2097 (2002).

[58] A. S. Fletcher, P. W. Shor, and M. Z. Win, Optimum quantum error recovery using semidefinite programming, Phys. Rev. A 75, 012338 (2007).

[59] C. Bény and O. Oreshkov, General Conditions for Approximate Quantum Error Correction and Near-Optimal Recovery Channels, Phys. Rev. Lett. 104, 120501 (2010).

[60] H. K. Ng and P. Mandayam, Simple approach to approximate quantum error correction based on the transpose channel, Phys. Rev. A 81, 062342 (2010).

[61] J. Tyson, Two-sided bounds on minimum-error quantum measurement, on the reversibility of quantum dynamics, and on maximum overlap using directional iterates, J. Math. Phys. 51, 092204 (2010).

[62] V. V. Albert, K. Noh, K. Duivenvoorden, D. J. Young, R. Brierley, P. Reinhold, C. Vuillot, L. Li, C. Shen, S. Girvin et al., Performance and structure of single-mode bosonic codes, Phys. Rev. A 97, 032346 (2018).

[63] S. Zhou and L. Jiang, An exact correspondence between the quantum Fisher information and the Bures metric, arXiv: 1910.08473 .

[64] S. Boyd and L. Vandenberghe, Convex Optimization (Cambridge University Press, Cambridge, 2004).

[65] H. Komiya, Elementary proof for Sion's minimax theorem, Kodai Math. J. 11, 5 (1988).

[66] M. do Rosário Grossinho and S. A. Tersian, An Introduction to Minimax Theorems and Their Applications to Differential Equations (Springer Science \& Business Media, New York, 2001), Vol. 52.

[67] E. Knill and R. Laflamme, Theory of quantum error-correcting codes, Phys. Rev. A 55, 900 (1997).

[68] C. Bény, Perturbative Quantum Error Correction, Phys. Rev. Lett. 107, 080501 (2011).

[69] L. Mirsky, Symmetric gauge functions and unitarily invariant norms, Quarterly J. Math. 11, 50 (1960). 